\title{
INTUITIONISTIC FUZZY ROUGH TOPSIS METHOD FOR ROBOT SELECTION USING EINSTEIN OPERATORS
}

\author{
ABBAS QADIR, MUHAMMAD NAEEM, SALEEEM ABDULLAH, AND NEJIB GHANMI
}

\begin{abstract}
Rough set and intuitionistic fuzzy set are very vital role in the decision making method for handling the uncertain and imprecise data of decision makers. The technique for order preference by similarity to ideal solution (TOPSIS) is very attractive method for solving the ranking and multi-criteria decision making (MCDM) problem. The primary goal of this paper is to introduce the Extended TOPSIS for industrial robot selection under intuitionistic fuzzy rough (IFR) information, where the weights of both, decision makers (DMs) and criteria are not-known. First, we develop Intuitionistic fuzzy rough (IFR) aggregation operators based on Einstein T-norm and T-conom, For this firstly we give the idea of intuitionistic fuzzy rough Einstein weighted averaging (IFREWA), intuitionistic fuzzy rough Einstein hybrid averaging (IFREHA) and intuitionistic fuzzy rough ordered weighted averaging (IFREOWA) aggregation operators. The fundamental properties of the proposed operators are described in detail. Furthermore to determine the unknown weights, a generalized distance measure are defined for IFRSs based on intuitionistic fuzzy rough entropy measure. Following that, the intuitionistic fuzzy rough information-based decision-making technique for multi-criteria group decision making (MCGDM) is developed, with all computing steps depicted in simplest form. For considering the conflicting attributes, our proposed model is more accurate and effective. Finally, an example of efficient industrial robot selection is presented to illustrate the feasibility of the proposed intuitionistic fuzzy rough decision support approaches, as well as a discussion of comparative outcomes, demonstrating that the results are feasible and reliable.
\end{abstract}

\section{INTRODUCTION}

Multi-criteria group decision making (MCGDM) has become extremely valuable in last few decades in the study of decision support systems $[1,2,3,4,5,6]$. The complexity of decision-making (DM) problems increases time to time in this world of global competition as the socio-economic framework becomes more dynamic. Therefore, making wise and successful decisions in this situation is very complicated for a single decision expert. In this real world, group DM models are often used to combine the opinions of team of experienced experts in order to generate highly accurate and optimal values. Hence, MCGDM have an excellent ability and systematic method for improving and evaluating various competing criteria in all aspect of DM in order to achieve more appropriate and possible DM outcomes. In DM issues, the knowledge based about even a fact is often unclear, making the decision-making task more complicated and ambiguous. To resolve this limitation, Zadeh [7] in 1965 first established the idea of fuzzy set (FS) by considering the membership degree. This concept has been investigated in a various real-world

Key words and phrases. Intuitionistic fuzzy rough set, Extended TOPSIS, Einstein Aggregation Operators, Decision Making, robot selection. 
problems, including clustering analysis [8] decision-making problems (DMPs) [9] and medical diagnosis [10]. Atanassov [11] developed the Intuitionistic Fuzzy Set (IFS) as an extension of the FS, and its constraint is that the sum of the degree of membership and the degree of non-membership will be less than or equal to one. IFS has been a hot research topic for researchers who examined its hybrid structure in a variety of ways. The idea of intuitionistic fuzzy weighted averaging (IFWA) aggregation operators was first investigated by $\mathrm{Xu}$ [12]. Xu and Yager [13] developed the definition of intuitionistic fuzzy weighted geometric (IFWG) aggregation operators. The graphical techniques for rating score and accuracy functions was created by Ali et al., [14]. He et al., [15] explored the concept of intuitionistic fuzzy neutral averaging operators (IFNAO). He et al., [16] invented the notions of geometric relationship averaging operator and proposed its implementation in DM. Also Atanassov [17] presented preference relation based on IVIF. Further Wan and Don [18] work on extension of best-worst method based on IFS. Zaho et al., [19] were the first to apply the generalized intuitionistic fuzzy weighted averaging (GIFWA), generalized intuitionistic fuzzy order weighted averaging (IFOWA), and generalized intuitionistic fuzzy hybrid averaging (GIFHA) operators to DM. However, it was discovered that these aggregation process was performed by using Archimedean Tnorms and T-conorm by operators. As an equivalent to algebraic product and sum, Einstein-based T-norm and T-conorm provides the best estimate for product and sum of intuitionistic fuzzy numbers (IFNs). Wang and Liu [20, 21] provided IF Einstein weighted averaging (IFEWA) and IF Einstein weighted geometric (IFEWG) operators by using the concept of Einstein operation.

After more outcomes," Pawlak [22] is credited with being the first to explore the prevalent idea of rough sets (RS) theories. This theory expanded on the classical set theory, which deals with ambiguous and imprecise information. Rough set analysis has advanced significantly in recent years, both in terms of practical applications and theoretical understanding. The idea of rough sets has been expanded in different ways by many researchers. The idea of fuzzy rough (FR) collection was first invented by Dubois and Prade [23] by utilizing fuzzy relations instead of crisp binary relation. Cornelis et al., [24] established a combined analysis of intuitionistic fuzzy rough set (IFRSs) applying the hybrid concept of IFS and RS as link between all these two theories. By using IFR approximation operators, Zhou and Wu [25] established a constrictive and axiomatic analysis. Zhou and $\mathrm{Wu}[26]$ created the idea of IFRS, rough intuitionistic fuzzy set and presented their constraining and axiomatic analysis in detail by introducing the idea of crisp and fuzzy approximation space. The IF relation was established by Bustince and Burillo [27]. Zhang et al., [28] utilized general IF relations to examine the general structure of IFRS based on the principle of two universes. Yun and Lee [29] used topology to establish some properties of IFR approximation operator based on IF relation. Many number of IFRSs extensions are examined, see [30, 31, 32, 33] for more information. In addition, Mehmood et al., [34] invented the interaction between the rough and IFS and also discussed its various aggregation operators.

Several decision-making processes are being proposed in the literature over the years, with techique for order preference by similarity to ideal solution (TOPSIS) being one of the most widely and efficiently used. To handle with MCGDM problems, Hwang and Yoon [35] addressed the TOPSIS. In DM problems, the best alternative is the one with the lowest distance from the positive ideal solution (PIS) 
and the highest distance from negative ideal solution (NIS). In [36] Chen discussed how to solve DM problems using TOPSIS under the FS. In recent decades, several researchers have become interested in TOPSIS and have applied it to real-world DM problems using various extended structures of FS [37] to [49] in the fields of decision sciences $[49,50,51]$. It's also worth noting that the current TOPSIS procedures [37] to [49] have the downside of requiring either DMs weights [45] or criteria weights $[41,48]$ or both $[37,39,48,49,50,51]$ to be known when solving DMPs. Some scholars assigned unknown weight details regarding DMs in which the parameters weights are known $[52,53]$.

Motivation: As for our information and based from the above review, no implementation of the MCGDM approach to a combination review of intuitionistic fuzzy set and rough sets using intuitionistic fuzzy Einstein averaging aggregation operators in intuitionistic fuzzy Rough setting in which the knowledge about DMs weights and criteria are completely unknown. MCGDM is used to demonstrate the efficiency of the established IFR Extended TOPSIS framework, which is focused on IFR Einstein averaging operators. We proposed the various average aggregation operators like intuitionistic fuzzy rough Einstein weighted averaging (IFREWA), intuitionistic fuzzy rough Einstein order weighted averaging (IFREOWA) and intuitionistic fuzzy rough Einstein hybrid averaging (IFREHA), by using the idea of Einstein T-norm and T-conorm for solving MCGDM. The main contribution of the proposed work are as following:

1) Using the Einstein T-norm and T-conorm to develop Einstein-aggregation operators for intuitionistic fuzzy rough information

2) We develop a new extended version of TOPSIS method by using the intuitionistic fuzzy rough Einstein aggregation information

3) The proposed techniques applied to industrial robot selection problems

4) The proposed decision techniques compared with previous decision making models and the comparative analysis show that the proposed method has more reliable and effective for ranking and decision making problems.

Throughout in this article, a novel Extended TOPSIS-based approach is developed to deal a problem with uncertain weight knowledge for both DMs and criteria weights, as well as to resolve the MCGDM problem even with all the weights have been computed. Ideal opinion (IO) which is closed to every DMs should be chosen for solving DM problems. Under proposed IFR Einstein average method, the ideal opinion is nominated in the presented procedure. To find the differences between two IFRSs, a generalized distance measure is defined. Generalized distance measures-based entropy measure is implemented in the presented IFR-Extended TOPSIS approach for solving MCGDM problems to find the criteria weights in the form of IFR information which are explained in this article. The fundamental principle for calculating criteria weights using the entropy measure is that the lower the entropy measure of a criterion between alternatives, the larger the weight should be applied on that criterion, but instead, the smaller weight should be applied on that criterion. A newer entropy measure for IFRSs is described as taking into consideration the value of its membership, non-membership grade and hesitancy degree in order to better measure the fuzziness of IFRSs, which is used to achieve the criteria weights in solving MCGDM problems with completely unknown weight information using the IFR entropy weight. 
The rest of this article is arranged as follows: Section 2, includes the essential principles of IFRSs that will be useful in following section. Section 3, exists the Einstein operation laws for IFRSs and concepts of Einstein averaging operators for IFRSs such as IFREWA, IFREOWA, (IFREHA), also discussed their properties. Section 4 , here in this section we proposed entropy measure and distance measures using IFRs information. Section 5, utilizing all these developed ideas we have presented the proposed Extended TOPSIS to resolve the uncertainty in MCGDM problems in this section. Section 6, exists numerical example for robot selection using the built MCGDM technique. Section 7 present comparison to other DM approaches. Section 8, draws a conclusion to the article.

List of Notation

\begin{tabular}{||l||l||}
\hline \hline Intuitionistic Fuzzy Set & IFS \\
\hline Intuitionistic Fuzzy Rough Set & IFRS \\
\hline Intuitionistic fuzzy rough Einstein weighted averaging & IFREWA \\
\hline Intuitionistic fuzzy rough Einstein ordered weighted averaging & IFREOWA \\
\hline Intuitionistic fuzzy rough Einstein hybrid weighted averaging & IFREHWA \\
\hline Multi-criteria group decision making & MCGDM \\
\hline Intuitionistic fuzzy Einstein weighted averaging & IFEWA \\
\hline Intuitionistic fuzzy Einstein weighted geometric & IFEWG \\
\hline intuitionistic fuzzy neutral averaging operators & IFNAO \\
\hline intuitionistic fuzzy rough value & IFRV \\
\hline
\end{tabular}

\section{PRELIMINARIES}

This section is considered for some fundamental concept for the remaining paper.

Definition 1. [25] Let $\Theta$ be a universe set and $\Upsilon \in \Theta \times \Theta$ are said to be crisp relation when the following properties hold;

(1) If $\left(p^{*}, p^{*}\right) \in \Upsilon$, for all $p^{*} \in \Theta$, Then $\Upsilon$ is (reflexive).

(2) If $p^{*}, d^{*} \in \Theta,\left(p^{*}, d^{*}\right) \in \Upsilon$ then $\left(d^{*}, p^{*}\right) \in \Upsilon$, $\Upsilon$ is (symmetric).

(3) If for all $p^{*}, d^{*}, e^{*} \in \Theta,\left(p^{*}, d^{*}\right) \in \Upsilon$ and $\left(d^{*}, e^{*}\right) \in \Upsilon$, then $\left(p^{*}, e^{*}\right) \in \Upsilon$. $\Upsilon$ is (transitive).

Definition 2. [25] Suppose $\Theta$ be a universe set and $\Upsilon \in \Theta \times \Theta$ be an arbitrary relation on set $\Theta$. Then defined a set valued mapping $\Upsilon^{*}: \Theta \longrightarrow C(\Theta)$ as:

$$
\Upsilon^{*}\left(p^{*}\right)=\left\{x \in \Theta \mid\left(p^{*}, x^{*}\right) \in \Theta\right\}, \text { for } p^{*} \in \Theta \text {. }
$$

where successor neighborhood is denoted by $\Upsilon^{*}\left(p^{*}\right)$ of an object $p^{*}$ w.r.t. $\Upsilon$. Then the pair $(\Theta, \Upsilon)$ as known as crisp approximation space. The lower and upper approximation for any $£ \subseteq \Theta$ w.r.t. approximation space denoted by $(\Theta, \Upsilon)$, are defined as follows:

$$
\begin{gathered}
\Upsilon(£)=\left\{p^{*} \in \Theta \mid \Upsilon^{*}\left(p^{*}\right) \subseteq £\right\}, \\
\bar{\Upsilon}(£)=\left\{p^{*} \in \Theta \mid \Upsilon^{*}\left(p^{*}\right) \cap £ \neq \phi\right\} .
\end{gathered}
$$

Then the rough set is represented by the pair $(\underline{\Upsilon}(£), \bar{\Upsilon}(£))$ and $\underline{\Upsilon}(£), \bar{\Upsilon}(£)$ : $C(\Theta) \longrightarrow C(\Theta)$ represents the lower and upper approximation operators.

Definition 3. [11] Suppose $\Theta$ be a universe set, then the structure

$$
\Upsilon=\left\{\left\langle p^{*}, \nu_{\Upsilon}\left(p^{*}\right), \eta_{\Upsilon}\left(p^{*}\right)\right\rangle \mid p^{*} \in \Theta\right\}
$$


are said to be intiuitionistic fuzzy set (IFS) where $\nu_{\Upsilon}: \Theta \longrightarrow[0,1]$ is called the membership degree and $\eta_{\Upsilon}: \Theta \longrightarrow[0,1]$ is non membership degree for each element $p^{*}$ belong to $\Upsilon$ satisfying the condition $0 \leq \nu_{\Upsilon}\left(p^{*}\right)+\eta_{\Upsilon}\left(p^{*}\right) \leq 1$. For simplicity the structure are represented by $\left(\nu_{\Upsilon}, \eta_{\Upsilon}\right)$.

Definition 4. [25] Consider a universal set $\Theta$ and $\Upsilon \in I F S(\Theta \times \Theta)$ is an IF relation. Then,

(1) For all $p^{*} \in \Theta$, if $\nu_{\Upsilon}\left(p^{*}, p^{*}\right)=1$ and $\eta_{\Upsilon}\left(p^{*}, p^{*}\right)=0$. Then $\Upsilon$ is called (reflexive).

(2) If for all $\left(p^{*}, d^{*}\right) \in \Theta \times \Theta, \nu_{\Upsilon}\left(p^{*}, d^{*}\right)=\nu_{\Upsilon}\left(d^{*}, p^{*}\right)$ and $\eta_{\Upsilon}\left(p^{*}, d^{*}\right)=$ $\eta_{\Upsilon}\left(d^{*}, p^{*}\right)$; Then $\Upsilon$ is (symmetric).

(3) If for all $\left(p^{*}, e^{*}\right) \in \Theta \times \Theta, \nu_{\Upsilon}\left(p^{*}, e^{*}\right) \geq \vee_{d^{*} \in \Theta}\left[\nu_{\Upsilon}\left(p^{*}, d^{*}\right) \wedge \nu_{\Upsilon}\left(d^{*}, e^{*}\right)\right]$ and $\eta_{\Upsilon}\left(p^{*}, e^{*}\right)=\wedge_{d^{*} \in \Theta}\left[\eta_{\Upsilon}\left(p^{*}, d^{*}\right) \wedge \eta_{\Upsilon}\left(d^{*}, e^{*}\right)\right]$. Then $\Upsilon$ is (transitive).

Definition 5. [34] Consider a fixed set $\Theta$, and $\Upsilon$ is any subset of a fixed set $\Theta$. Then, $\Upsilon \in I F S(\Theta \times \Theta)$ is said to be an IF relation. The pair $(\Theta, \Upsilon)$ is said to an intuitionistic fuzzy approximation space (IFAS). The upper and lower approximations of $£$ in term of $\operatorname{IFAS}(\Theta, \Upsilon)$, for any $£ \subseteq I F S(\Theta)$ are two $\operatorname{IFSs} \bar{\Upsilon}(£)$ and $\Upsilon(£)$, which are defined as follows:

$$
\begin{aligned}
& \bar{\Upsilon}(£)=\left\{\left\langle p^{*}, \nu_{\bar{\Upsilon}(£)}\left(p^{*}\right), \eta_{\bar{\Upsilon}(£)}\left(p^{*}\right) \mid p^{*} \in \Theta\right\rangle\right\}, \\
& \underline{\Upsilon}(£)=\left\{\left\langle p^{*}, \nu_{\underline{\Upsilon}(£)}\left(p^{*}\right), \eta_{\underline{\Upsilon}(£)}\left(p^{*}\right) \mid p^{*} \in \Theta\right\rangle\right\},
\end{aligned}
$$

where $\nu_{\bar{\Upsilon}(£)}\left(p^{*}\right)=\bigvee_{d^{*} \in \Theta}\left[\nu_{\Upsilon}\left(p^{*}, d^{*}\right) \vee \nu_{£}\left(d^{*}\right)\right]$, and $\eta_{\bar{\Upsilon}(£)}\left(p^{*}\right)=\bigwedge_{d^{*} \in \Theta}\left[\eta_{\Upsilon}\left(p^{*}, d^{*}\right) \wedge\right.$

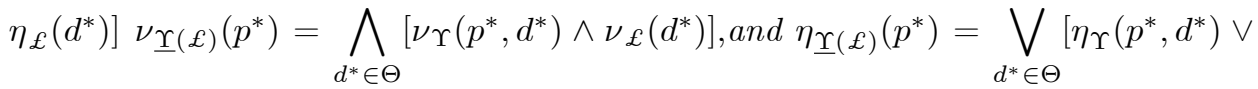

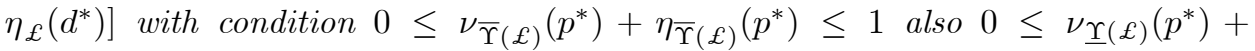
$\eta_{\Upsilon(£)}\left(p^{*}\right) \leq 1$. Where $\bar{\Upsilon}(£)$ and $\underline{\Upsilon}(£)$ IFSs, so $\bar{\Upsilon}(£), \underline{\Upsilon}(£): \operatorname{IFS}(\Theta) \longrightarrow \operatorname{IFS}(\Theta)$ is upper and lower approximation operators. Then the pair

$(2.6) \Upsilon(£)=(\underline{\Upsilon}(£), \bar{\Upsilon}(£))$

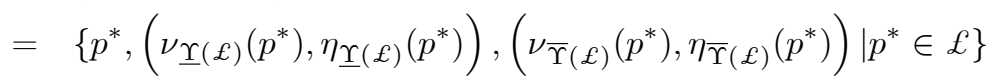

are said to be IF rough set. Shortly it is represented by $\Upsilon(\kappa)=((\underline{\nu}, \eta),(\bar{\nu}, \bar{\eta}))$. See example [34]

Definition 6. [34] Suppose $\Upsilon(£)=(\underline{\Upsilon}(£), \bar{\Upsilon}(£))=((\underline{\nu}, \underline{\eta}),(\bar{\nu}, \bar{\eta}))$ be a intuitionistic fuzzy rough value (IFRV). Then the score and accuracy function is given by,

$$
\text { S }(\Upsilon(£))=\frac{1}{4}(2+\underline{\nu}+\bar{\nu}-\underline{\eta}-\bar{\eta}), \text { where }(S(\Upsilon(£)) \in[1,0] \text {. }
$$

$A_{\mathbb{C}}(\Upsilon(£))=\frac{1}{4}(\underline{\nu}+\bar{\nu}+\underline{\eta}+\bar{\eta})$, where $A_{\mathbb{C}}(\Upsilon(£)) \in[1,0]$. 
Definition 7. [34] Let $\Upsilon(£)=((\underline{\nu}, \underline{\eta}),(\bar{\nu}, \bar{\eta}))$ be the IFRS, Then the degree of hesitancy IFRS is determined by,

$$
\begin{aligned}
& \Xi \Upsilon(£)=(\Xi \Upsilon(£), \Xi \bar{\Upsilon}(£)) \\
& \Xi \Upsilon(£)=1-\underline{\nu}-\bar{\eta} \\
& \Xi \bar{\Upsilon}(£)=1-\bar{\nu}-\overline{\bar{\eta}}
\end{aligned}
$$

\section{Einstein Operations FOR intuitionistic FUZZy ROUGH SETS}

Here we present the Einstein operations and fundamental properties of IFRSs by taking the ideas of [56]. If T-norm and T-conorm are represented by $\check{T}$ and $\hat{S}$. Then Einstein product and sum are expressed by $\check{T}_{\varsigma}$ and $\hat{S}_{\varsigma}$ respectively. The Einstein sum and product are equivalent to the generalized union and intersection of two IFRSs, which are defined as follows:

$$
\begin{aligned}
& \Upsilon\left(£_{1}\right) \oplus_{\xi} \Upsilon\left(£_{2}\right)=\left\langle\begin{array}{c}
p^{*}, \hat{S}_{\varsigma}\left(\underline{\nu}_{1}\left(p^{*}\right), \underline{\nu}_{2}\left(p^{*}\right)\right), \check{T}_{\varsigma}\left(\underline{\eta}_{1}\left(p^{*}\right), \underline{\eta}_{2}\left(p^{*}\right)\right), \\
\hat{S}_{\varsigma}\left(\bar{\nu}_{1}\left(p^{*}\right), \bar{\nu}_{2}\left(p^{*}\right)\right), \check{T}_{\varsigma}\left(\bar{\eta}_{1}\left(p^{*}\right), \bar{\eta}_{2}\left(p^{*}\right)\right) p^{*} \in \Theta
\end{array}\right\rangle \\
& \Upsilon\left(£_{1}\right) \otimes_{\xi} \Upsilon\left(£_{2}\right)=\left\langle\begin{array}{c}
p^{*}, \check{T}_{\varsigma}\left(\underline{\nu}_{1}\left(p^{*}\right), \underline{\nu}_{2}\left(p^{*}\right)\right), \hat{S}_{\varsigma}\left(\underline{\eta}_{1}\left(p^{*}\right), \underline{\eta}_{2}\left(p^{*}\right)\right), \\
\check{T}_{\varsigma}\left(\bar{\nu}_{1}\left(p^{*}\right), \bar{\nu}_{2}\left(p^{*}\right)\right), \hat{S}_{\varsigma}\left(\bar{\eta}_{1}\left(p^{*}\right), \bar{\eta}_{2}\left(p^{*}\right)\right) p^{*} \in \Theta
\end{array}\right\rangle
\end{aligned}
$$

Further more, we can derive the following forms:

Definition 8. Let us we have two IFRSs $\Upsilon\left(£_{1}\right)=\left(\left(\underline{\nu}_{1}, \underline{\eta}_{1}\right),\left(\bar{\nu}_{1}, \bar{\eta}_{1}\right)\right)$ and $\Upsilon\left(£_{2}\right)=$ $\left(\left(\underline{\nu}_{2}, \underline{\eta}_{2}\right),\left(\bar{\nu}_{2}, \bar{\eta}_{2}\right)\right)$. Then, the Einstein basic operation are as follows:

(1)

$$
\Upsilon\left(£_{1}\right) \otimes_{\xi} \Upsilon\left(£_{2}\right)=\left[\begin{array}{c}
\left(\frac{\underline{\nu}_{1} \cdot \underline{\nu}_{2}}{1+\left(1-\underline{\nu}_{1}\right) \cdot\left(1-\underline{\nu}_{2}\right)}, \frac{\underline{\eta}_{1}+\underline{\eta}_{2}}{1+\underline{\eta}_{1} \cdot \eta_{2}}\right), \\
\left(\frac{\bar{\nu}_{1} \cdot \bar{\nu}_{2}}{1+\left(1-\bar{\nu}_{1}\right) \cdot\left(1-\bar{\nu}_{2}\right)}, \frac{\bar{\eta}_{1}+\overline{\bar{\eta}}_{2}}{1+\bar{\eta}_{1}+\bar{\eta}_{2}}\right)
\end{array}\right],
$$

$$
\Upsilon\left(£_{1}\right) \oplus_{\xi} \Upsilon\left(£_{2}\right)=\left[\begin{array}{c}
\left(\frac{\underline{\nu}_{1}+\underline{\nu}_{2}}{1+\underline{\nu}_{1} \cdot \underline{\nu}_{2}}, \frac{\underline{\eta}_{1} \cdot \underline{\eta}_{2}}{1+\left(1-\underline{\eta}_{1}\right) \cdot\left(1-\underline{\eta}_{2}\right)}\right), \\
\left(\frac{\bar{\nu}_{1}+\bar{\nu}_{2}}{1+\bar{\nu}_{1} \cdot \bar{\nu}_{2}}, \frac{\bar{\eta}_{1}+\bar{\eta}_{2}}{1+\left(1-\bar{\eta}_{1}\right) \cdot\left(1-\bar{\eta}_{2}\right)}\right)
\end{array}\right]
$$

$$
\left.\lambda \Upsilon\left(£_{1}\right)=\left(\begin{array}{l}
\left(\frac{\left[1+\underline{\nu}_{1}\right]^{\lambda}-\left[1-\underline{\nu}_{1}\right]^{\lambda}}{\left[1+\underline{\nu}_{1}\right]^{\lambda}+\left[1-\underline{\nu}_{1}\right]^{\lambda}}, \frac{2\left[\underline{\eta}_{1}\right]^{\lambda}}{\left[2-\underline{\eta}_{1}\right]^{\lambda}+\left[\underline{\eta}_{1}\right]^{\lambda}}\right. \\
\left(\frac{\left[1+\bar{\nu}_{1}\right]^{\lambda}-\left[1-\bar{\nu}_{1}\right]^{\lambda}}{\left[1+\bar{\nu}_{1}\right]^{\lambda}+\left[1-\bar{\nu}_{1}\right]^{\lambda}}, \frac{\left.2 \bar{\eta}_{1}\right]^{\lambda}}{\left[2-\bar{\eta}_{1}\right]^{\lambda}+\left[\bar{\eta}_{1}\right]^{\lambda}}\right.
\end{array}\right)\right)
$$

$$
\left.\left(\Upsilon\left(£_{1}\right)\right)^{\lambda}=\left(\begin{array}{c}
\left(\frac{2\left[\underline{\nu}_{1}\right]^{\lambda}}{\left[2-\underline{\nu}_{1}\right]^{\lambda}+\left[\underline{\nu}_{1}\right]^{\lambda}}, \frac{\left[1+\underline{\eta}_{1}\right]^{\lambda}-\left[1-\underline{\eta}_{1}\right]^{\lambda}}{\left[1+\underline{\eta}_{1}\right]^{\lambda}+\left[1-\bar{\eta}_{1}\right]^{\lambda}}\right. \\
\left(\frac{2\left[\bar{\nu}_{1}\right]^{\lambda}}{\left[2-\bar{\nu}_{1}\right]^{\lambda}+\left[\bar{\nu}_{1}\right]^{\lambda}}, \frac{\left[1+\bar{\eta}_{1}\right]^{\lambda}-\left[1-\bar{\eta}_{1}\right]^{\lambda}}{\left[1+\bar{\eta}_{1}\right]^{\lambda}+\left[1-\bar{\eta}_{1}\right]^{\lambda}}\right.
\end{array}\right)\right)
$$




\subsection{INTUITIONISTIC FUZZY ROUGH EINSTEIN WEIGHTED AV-}

ERAGING OPERATOR. This part we will initiate the IFREWA operator. Also, certain fundamental properties will be debated.

Definition 9. Let us we have the collection $\Upsilon\left(£_{\imath}\right)=\left(\underline{\Upsilon}\left(£_{\imath}\right), \bar{\Upsilon}\left(£_{\imath}\right)\right)(\imath=1,2, \ldots, n)$ of IFRVs having weight vector $b=\left(b_{1}, b_{2}, \ldots, b_{n}\right)^{T}$ with condition $\sum_{\imath=1}^{n} b_{\imath}=1$ and $0 \leq b_{\imath} \leq 1$. Then (IFREWA) is defined as:

$$
\left.\left.\operatorname{IFREWA} A\left(£_{1}\right), \Upsilon\left(£_{2}\right), \ldots, \Upsilon\left(£_{n}\right)\right)\right)=\left(\oplus_{\imath=1}^{n} b_{\imath} \underline{\Upsilon}\left(£_{\imath}\right), \oplus_{\imath=1}^{n} b_{\imath} \bar{\Upsilon}\left(£_{\imath}\right)\right)
$$

Theorem 1 shows the aggregated results for the IFREWA operator based on the above definition.

Theorem 1. Let us we have the collection $\Upsilon\left(£_{\imath}\right)=\left(\underline{\Upsilon}\left(£_{\imath}\right), \bar{\Upsilon}\left(£_{\imath}\right)\right)(\imath=1,2, \ldots, n)$ of IFRVs having weights vectors $b=\left(b_{1}, b_{2}, \ldots, b_{n}\right)^{T}$ with condition $\sum_{\imath=1}^{n} b_{\imath}=1$ and $0 \leq b_{\imath} \leq 1$. The IFREWA operator is defined as follows:

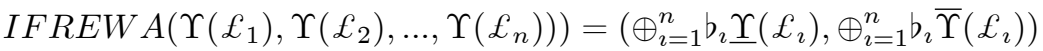

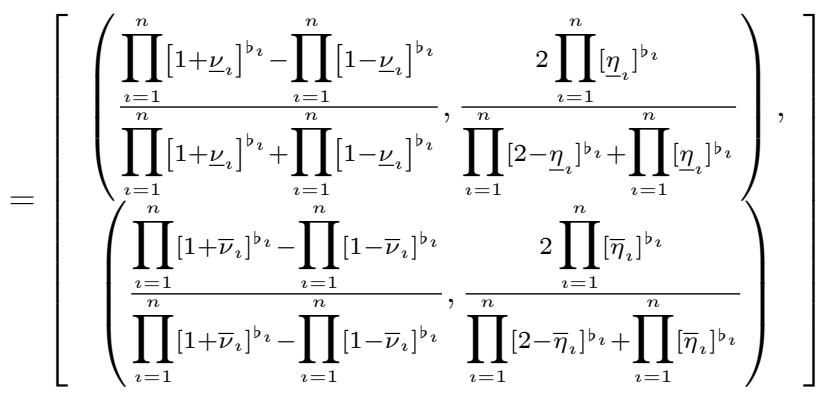

Proof. To obtain the above result for this we use mathematical induction. As by defined Einstein operation law,

$$
\begin{gathered}
\Upsilon\left(£_{1}\right) \oplus \Upsilon\left(£_{2}\right)=\left[\left(\underline{\Upsilon}\left(£_{1}\right) \oplus \underline{\Upsilon}\left(£_{2}\right)\right),\left(\bar{\Upsilon}\left(£_{1}\right) \oplus \bar{\Upsilon}\left(£_{2}\right)\right)\right] \\
=\left[\begin{array}{c}
\left(\frac{\underline{\nu}_{1}+\underline{\nu}_{2}}{1+\underline{\nu}_{1} \cdot \underline{\nu}_{2}}, \frac{\underline{\eta}_{1} \cdot \underline{\eta}_{2}}{1+\left(1-\underline{\eta}_{1}\right) \cdot\left(1-\underline{\eta}_{2}\right)}\right), \\
\left(\frac{\bar{\nu}_{1} \cdot \bar{\nu}_{2}}{1+\bar{\nu}_{1} \cdot \bar{\nu}_{2}}, \frac{\bar{\eta}_{1}+\bar{\eta}_{2}}{1+\left(1-\bar{\eta}_{1}\right) \cdot\left(1-\bar{\eta}_{2}\right)}\right)
\end{array}\right]
\end{gathered}
$$

and

$$
\begin{gathered}
\lambda \Upsilon\left(£_{1}\right)=\left(\lambda \underline{\Upsilon}\left(£_{1}\right), \lambda \bar{\Upsilon}\left(£_{1}\right)\right) \\
=\left[\begin{array}{c}
\left(\frac{\left[1+\underline{\nu}_{1}\right]^{\lambda}-\left[1-\underline{\nu}_{1}\right]^{\lambda}}{\left[1+\underline{\nu}_{1}\right]^{\lambda}+\left[1-\underline{\nu}_{1}\right]^{\lambda}}, \frac{2\left[\underline{\eta}_{1}\right]^{\lambda}}{\left[2-\underline{\eta}_{1}\right]^{\lambda}+\left[\underline{\eta}_{1}\right]^{\lambda}}\right), \\
\left(\frac{\left[1+\bar{\nu}_{1}\right]^{\lambda}-\left[1-\bar{\nu}_{1}\right]^{\lambda}}{\left[1+\bar{\nu}_{1}\right]^{\lambda}+\left[1-\bar{\nu}_{1}\right]^{\lambda}}, \frac{2\left[\bar{\eta}_{1}\right]^{\lambda}}{\left[2-\bar{\eta}_{1}\right]^{\lambda}+\left[\bar{\eta}_{1}\right]^{\lambda}}\right)
\end{array}\right]
\end{gathered}
$$

Now for $n=2$, then

$$
\operatorname{IFREWA}\left(\Upsilon\left(£_{1}\right), \Upsilon\left(£_{2}\right)\right)=\left[\oplus_{\imath=1}^{2} b_{\imath} \underline{\Upsilon}\left(£_{\imath}\right), \oplus_{\imath=1}^{2} b_{\imath} \bar{\Upsilon}\left(£_{\imath}\right)\right]
$$




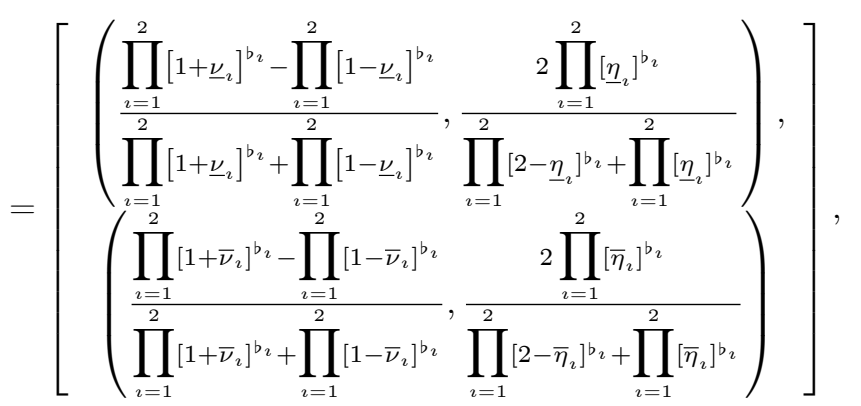

Now we check the result for $n=k$.

$$
\begin{aligned}
& \operatorname{IFREWA}\left(\Upsilon\left(£_{1}\right), \Upsilon\left(£_{2}\right), \ldots, \Upsilon\left(£_{k}\right)\right)
\end{aligned}
$$

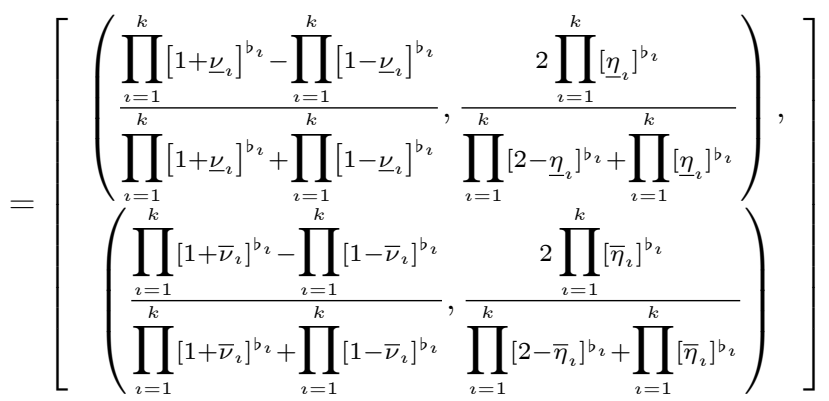

Now for $n=k+1$, we have

$$
\begin{aligned}
& \text { IFREW A [( } \left.\left.\left(£_{1}\right), \Upsilon\left(£_{2}\right), \ldots, \Upsilon\left(£_{k}\right)\right), \Upsilon\left(£_{k+1}\right)\right]
\end{aligned}
$$

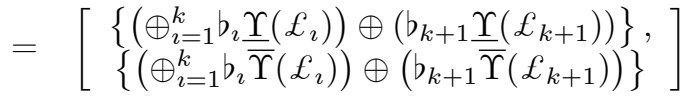

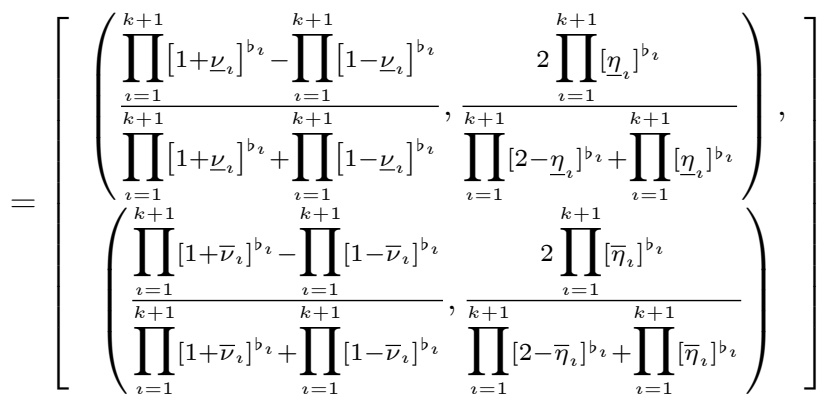

Now we conclude that the following result is true for $n=k+1$. The following result is true for all $n \geq 1$. From above results $\underline{\Upsilon}(£)$ and $\bar{\Upsilon}(£)$ are IFRVs. So $\oplus_{\imath=1}^{n} b_{\imath} \Upsilon\left(£_{\imath}\right)$ and $\oplus_{\imath=1}^{n} b_{\imath} \bar{\Upsilon}\left(£_{\imath}\right)$ are also IFRVs. Therefore IFREWA $\left(\Upsilon\left(£_{1}\right), \Upsilon\left(£_{2}\right), \ldots, \Upsilon\left(£_{n}\right)\right)$ is also a IFRV under IFAS $(\Theta, \Upsilon)$.

Theorem 2. Let us we have a collection $\Upsilon\left(£_{\imath}\right)=\left(\left(\underline{\nu}_{\imath}, \underline{\eta}_{\imath}\right),\left(\bar{\nu}_{\imath}, \bar{\eta}_{\imath}\right)\right)(\imath=1,2, . ., n)$ of IFRVs having weight vectors $b=\left(b_{1}, b_{2}, \ldots, b_{n}\right)^{T}$ with condition $\sum_{\imath=1}^{n} b_{\imath}=1$ and $0 \leq b_{\imath} \leq 1$. Then their are some essential properties of IFREWA operator, which are as follow:

(i). (Idempotency): If $\Upsilon\left(£_{\imath}\right)=C(K) \forall \imath=1,2, . ., n$ and $C(K)=(\underline{C}(K), \bar{C}(K))=$ $((\underline{g}, \underline{h}),(\bar{g}, \bar{h}))$. Then

$$
\operatorname{IFREWA}\left(\Upsilon\left(£_{1}\right), \Upsilon\left(£_{2}\right), \ldots, \Upsilon\left(£_{n}\right)\right)=C(K)
$$


(ii). (Boundedness): Suppose $(\Upsilon(£))^{-}=(\Upsilon(£))^{\inf }=\left(\inf _{\imath} \Upsilon\left(£_{\imath}\right), \sup \bar{\Upsilon}\left(£_{\imath}\right)\right)$ and $(\Upsilon(£))^{+}=(\Upsilon(£))^{\sup }=\left(\sup _{\imath} \Upsilon\left(£_{\imath}\right), \inf _{\imath} \bar{\Upsilon}\left(£_{\imath}\right)\right)$. Then

$$
(\Upsilon(£))^{-} \leq \operatorname{IFREWA} A\left(\Upsilon\left(£_{1}\right), \Upsilon\left(£_{2}\right), \ldots, \Upsilon\left(£_{n}\right)\right) \leq(\Upsilon(£))^{+} .
$$

(iii). (Monotonicity): Suppose we have another collection $C\left(L_{\imath}\right)=\left(\underline{C}\left(L_{\imath}\right), \bar{C}\left(L_{\imath}\right)\right)(\imath=$ $1,2, \ldots n)$ of IFRVs such that $\underline{C}\left(L_{\imath}\right) \leq \underline{\Upsilon}\left(£_{\imath}\right)$ and $\bar{C}\left(L_{\imath}\right) \leq \bar{\Upsilon}\left(£_{\imath}\right)$. Then

$$
\operatorname{IFREWA}\left(C\left(L_{1}\right), C\left(L_{2}\right), \ldots, C\left(L_{n}\right)\right) \leq \operatorname{IFREWA}\left(\Upsilon\left(£_{1}\right), \Upsilon\left(£_{2}\right), \ldots, \Upsilon\left(£_{n}\right)\right)
$$

Proof. (i). (Idempotency). For all $(\imath=1,2, \ldots n) \Upsilon\left(£_{\imath}\right)=C(K)$, where $C(K)=$ $(\underline{C}(K), \bar{C}(K))=((\underline{g}, \underline{h}),(\bar{g}, \bar{h}))$.

$$
\begin{aligned}
& \operatorname{IFREWA}\left(\Upsilon\left(£_{1}\right), \Upsilon\left(£_{2}\right), \ldots, \Upsilon\left(£_{n}\right)\right)=\left[\oplus_{\imath=1}^{n} b_{\imath} \underline{\Upsilon}\left(£_{\imath}\right), \oplus_{\imath=1}^{n} b_{\imath} \bar{\Upsilon}\left(£_{\imath}\right)\right]
\end{aligned}
$$

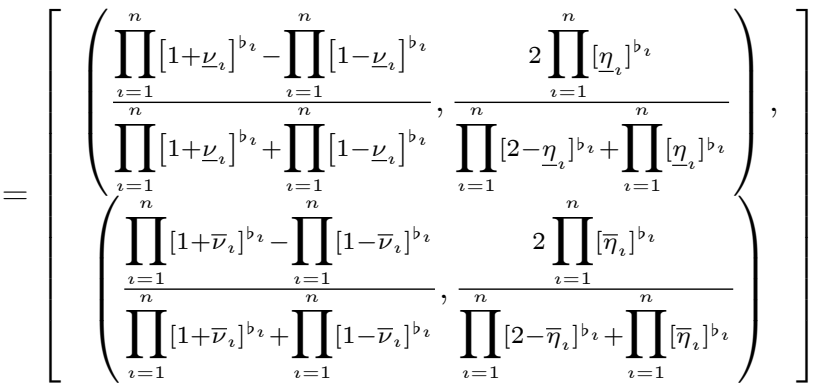

$\forall \imath, \Upsilon\left(£_{\imath}\right)=C(K)=(\underline{C}(K), \bar{C}(K))=((\underline{g}, \underline{h}),(\bar{g}, \bar{h}))$, Hence,

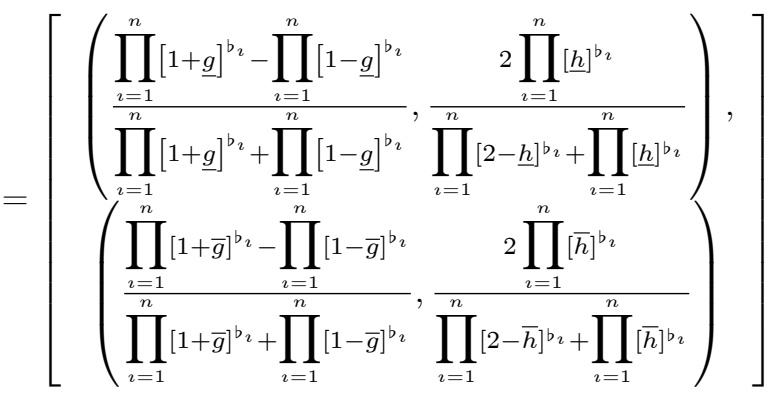

$$
\begin{aligned}
& =\left[\begin{array}{c}
\left(\frac{[1+\underline{g}]-[1-\underline{g}]}{[1+\underline{g}]+[1-\underline{g}]}, \frac{2[\underline{h}]}{[2-\underline{h}]+[\underline{h}]}\right), \\
\left(\frac{[1+\bar{g}]-[1-\bar{g}]}{[1+\bar{g}]+[1-\bar{g}]}, \frac{2[\bar{h}]}{[2-\bar{h}]+[\bar{h}]}\right)
\end{array}\right] \\
& =(\underline{C}(K), \bar{C}(K))=C(K)
\end{aligned}
$$

Hence,

$$
\operatorname{IFREWA}\left(\Upsilon\left(£_{1}\right), \ldots, \Upsilon\left(£_{n}\right)\right)=C(K)
$$

(ii) (Boundedness). As

$$
\begin{aligned}
& (\underline{\Upsilon}(£))^{\inf }=\left[\left(\inf _{\imath}\left\{\underline{\nu}_{\imath}\right\}, \sup _{\imath}\left\{\underline{\eta}_{\imath}\right\}\right),\left(\inf _{\imath}\left\{\bar{\nu}_{\imath}\right\}, \sup _{\imath}\left\{\bar{\eta}_{\imath}\right\}\right)\right], \\
& (\underline{\Upsilon}(£))^{\sup }=\left[\left(\sup _{\imath}\left\{\underline{\nu}_{\imath}\right\}, \inf _{\imath}\left\{\underline{\eta}_{\imath}\right\}\right),\left(\sup _{\imath}\left\{\bar{\nu}_{\imath}\right\}, \inf _{\imath}\left\{\bar{\eta}_{\imath}\right\}\right)\right],
\end{aligned}
$$


and

To prove that

$$
\Upsilon\left(£_{\imath}\right)=\left[\left(\underline{\nu}_{\imath}, \underline{\eta}_{\imath}\right),\left(\bar{\nu}_{\imath}, \bar{\eta}_{\imath}\right)\right] .
$$

$$
(\Upsilon(£))^{\inf } \leq I F R E W A\left(\Upsilon\left(£_{1}\right), \Upsilon\left(£_{2}\right), \ldots, \Upsilon\left(£_{n}\right)\right) \leq(\Upsilon(£))^{\text {sup }} .
$$

Let $f(c)=\frac{1-c}{1+c}, r \in[0,1]$; then $f(c)^{\prime}=\left[\frac{1-c}{1+c}\right]^{\prime}=\frac{-2}{(1+c)^{2}}<0$; thus the function $f(c)$ is decreasing. Since $\left(\underline{\nu}_{\imath}\right)^{\text {inf }} \leq \underline{\nu}_{\imath} \leq\left(\underline{\nu}_{\imath}\right)^{\text {sup }} \forall \imath$, then $f\left(\left(\underline{\nu}_{\imath}\right)^{\text {sup }}\right) \leq f\left(\underline{\nu}_{\imath}\right) \leq f\left(\left(\underline{\nu}_{\imath}\right)^{\text {inf }}\right)$, $\forall \imath$, that is. $\frac{1-\left(\underline{\nu}_{2}\right)^{\text {sup }}}{1+\left(\underline{\nu}_{\imath}\right)^{\text {sup }}} \leq \frac{1-\left(\underline{\nu}_{\imath}\right)}{1+\left(\underline{\nu}_{\imath}\right)} \leq \frac{1-\left(\underline{\nu}_{\imath}\right)^{\text {inf }}}{1+\left(\underline{\nu}_{\imath}\right)^{\text {inf }}},(\imath=1,2, \ldots, n)$. Let $b=\left(b_{1}, b_{2}, \ldots, b_{n}\right)^{T}$ be weighted vector of $\underline{\Upsilon}\left(£_{\imath}\right)(\imath=1,2, \ldots, n)$ with $\sum_{\imath=1}^{n} b_{\imath}=1$ such that $b_{\imath} \in[0,1]$. Then, $\forall \imath$, we have

$$
\begin{gathered}
\left(\frac{1-\left(\underline{\nu}_{\imath}\right)^{\sup }}{1+\left(\underline{\nu}_{\imath}\right)^{\sup }}\right)^{b_{\imath}} \leq\left(\frac{1-\left(\underline{\nu}_{\imath}\right)}{1+\left(\underline{\nu}_{\imath}\right)}\right)^{b_{\imath}} \leq\left(\frac{1-\left(\underline{\nu}_{\imath}\right)^{\inf }}{1+\left(\underline{\nu}_{\imath}\right)^{\inf }}\right)^{b_{\imath}}= \\
\prod_{\imath=1}^{n}\left(\frac{1-\left(\underline{\nu}_{\imath}\right)^{\sup }}{1+\left(\underline{\nu}_{\imath}\right)^{\sup }}\right)^{b_{\imath}} \leq \prod_{\imath=1}^{n}\left(\frac{1-\left(\underline{\nu}_{\imath}\right)}{1+\left(\underline{\nu}_{\imath}\right)}\right)^{b_{\imath}} \leq \prod_{\imath=1}^{n}\left(\frac{1-\left(\underline{\nu}_{\imath}\right)^{\inf }}{1+\left(\underline{\nu}_{\imath}\right)^{\inf }}\right)^{b_{\imath}}
\end{gathered}
$$

if and only if

$$
\sum_{\imath=1}^{n}\left(\frac{1-\left(\underline{\nu}_{\imath}\right)^{\mathrm{sup}}}{1+\left(\underline{\nu}_{\imath}\right)^{\sup }}\right)^{b_{\imath}} \leq \prod_{\imath=1}^{n}\left(\frac{1-\left(\underline{\nu}_{\imath}\right)}{1+\left(\underline{\nu}_{\imath}\right)}\right)^{b_{\imath}} \leq \sum_{\imath=1}^{n}\left(\frac{1-\left(\underline{\nu}_{\imath}\right)^{\inf }}{1+\left(\underline{\nu}_{\imath}\right)^{\inf }}\right)^{b_{\imath}}
$$

if and only if

$$
\left(\frac{1-\left(\underline{\nu}_{\imath}\right)^{\sup }}{1+\left(\underline{\nu}_{\imath}\right)^{\sup }}\right)^{b_{\imath}} \leq \prod_{\imath=1}^{n}\left(\frac{1-\left(\underline{\nu}_{\imath}\right)}{1+\left(\underline{\nu}_{\imath}\right)}\right)^{b_{\imath}} \leq\left(\frac{1-\left(\underline{\nu}_{\imath}\right)^{\text {inf }}}{1+\left(\underline{\nu}_{\imath}\right)^{\text {inf }}}\right)^{b_{\imath}}
$$

if and only if

$$
\left(\frac{2}{1+\left(\underline{\nu}_{\imath}\right)^{\sup }}\right) \leq 1+\prod_{\imath=1}^{n}\left(\frac{1-\left(\underline{\nu}_{\imath}\right)}{1+\left(\underline{\nu}_{\imath}\right)}\right)^{b_{\imath}} \leq\left(\frac{2}{1+\left(\underline{\nu}_{\imath}\right)^{\mathrm{inf}}}\right)
$$

if and only if

$$
\left(\frac{1+\left(\underline{\nu}_{\imath}\right)^{\mathrm{inf}}}{2}\right) \leq \frac{1}{1+\prod_{\imath=1}^{n}\left(\frac{1-\left(\underline{\nu}_{\imath}\right)}{1+\left(\underline{\nu}_{\imath}\right)}\right)^{b_{\imath}}} \leq\left(\frac{1+\left(\underline{\nu}_{\imath}\right)^{\text {sup }}}{2}\right)
$$

if and only if

$$
\left(1+\left(\underline{\nu}_{\imath}\right)^{\mathrm{inf}}\right) \leq \frac{2}{1+\prod_{\imath=1}^{n}\left(\frac{1-\left(\underline{\nu}_{\imath}\right)}{1+\left(\underline{\underline{\nu}}_{\imath}\right)}\right)^{b_{\imath}}} \leq\left(1+\left(\underline{\nu}_{\imath}\right)^{\mathrm{sup}}\right)
$$

implies that

$$
\left(\left(\underline{\nu}_{\imath}\right)^{\mathrm{inf}}\right) \leq \frac{2}{1+\prod_{\imath=1}^{n}\left(\frac{1-\left(\underline{\nu}_{\imath}\right)}{1+\left(\underline{\nu}_{\imath}\right)}\right)^{\mathrm{b}_{\imath}}}-1 \leq\left(\left(\underline{\nu}_{\imath}\right)^{\mathrm{sup}}\right)
$$


which implies that

$$
\left(\left(\underline{\nu}_{\imath}\right)^{\inf }\right) \leq \frac{\prod_{\imath=1}^{n}\left(1+\left(\underline{\nu}_{\imath}\right)\right)^{b_{\imath}}-\prod_{\imath=1}^{n}\left(1-\left(\underline{\nu}_{\imath}\right)\right)^{b_{\imath}}}{\prod_{\imath=1}^{n}\left(1+\left(\underline{\nu}_{\imath}\right)\right)^{b_{\imath}}+\prod_{\imath=1}^{n}\left(1-\left(\underline{\nu}_{\imath}\right)\right)^{b_{\imath}}} \leq\left(\left(\underline{\nu}_{\imath}\right)^{\sup }\right) .
$$

Now let we have another function $g(s)=\frac{2-s}{s}, s \in(0,1]$; then the function $g(s)^{\prime}=$ $\frac{-2}{s^{2}}<0$, is decreasing on interval $(0,1]$. Since $\left(\underline{\eta}_{\imath}\right)^{\text {sup }} \leq \underline{\eta}_{\imath} \leq\left(\underline{\eta}_{\imath}\right)^{\text {inf }}$, for all $\imath$, where $0<\left(\underline{\eta}_{\imath}\right)^{\text {sup }}$, then $g\left(\left(\underline{\eta}_{\imath}\right)^{\text {inf }}\right) \leq g\left(\underline{\eta}_{\imath}\right) \leq g\left(\left(\underline{\eta}_{\imath}\right)^{\text {sup }}\right) \forall \imath$. that is,

$$
\frac{2-\left(\underline{\eta}_{\imath}\right)^{\inf }}{\left(\underline{\eta}_{\imath}\right)^{\text {inf }}} \leq \frac{2-\underline{\eta}_{\imath}}{\underline{\eta}_{\imath}} \leq \frac{2-\left(\underline{\eta}_{\imath}\right)^{\text {sup }}}{\left(\underline{\eta}_{\imath}\right)^{\sup }},(\imath=1,2, \ldots, n) .
$$

Let $b=\left(b_{1}, b_{2}, \ldots, b_{n}\right)^{T}$ be weighted vector of $\Upsilon\left(£_{\imath}\right)(\imath=1,2, \ldots, n)$ with condition $\sum_{\imath=1}^{n} b_{\imath}=1$ such that $b_{\imath} \in[0,1]$. Then for all $\imath$, we have

$$
\left(\frac{2-\left(\underline{\eta}_{\imath}\right)^{\text {inf }}}{\left(\underline{\eta}_{\imath}\right)^{\text {inf }}}\right)^{b_{\imath}} \leq\left(\frac{2-\underline{\eta}_{\imath}}{\underline{\eta}_{\imath}}\right)^{b_{\imath}} \leq\left(\frac{2-\left(\underline{\eta}_{\imath}\right)^{\text {sup }}}{\left(\underline{\eta}_{\imath}\right)^{\text {sup }}}\right)^{b_{\imath}} .
$$

Thus,

$$
\prod_{\imath=1}^{n}\left(\frac{2-\left(\underline{\eta}_{\imath}\right)^{\text {inf }}}{\left(\underline{\eta}_{\imath}\right)^{\text {inf }}}\right)^{b_{\imath}} \leq \prod_{\imath=1}^{n}\left(\frac{2-\underline{\eta}_{\imath}}{\underline{\eta}_{\imath}}\right)^{b_{\imath}} \leq \prod_{\imath=1}^{n}\left(\frac{2-\left(\underline{\eta}_{\imath}\right)^{\text {sup }}}{\left(\underline{\eta}_{\imath}\right)^{\text {sup }}}\right)^{b_{\imath}}
$$

iff

$$
\sum_{\imath=1}^{n}\left(\frac{2-\left(\underline{\eta}_{\imath}\right)^{\text {inf }}}{\left(\underline{\eta}_{\imath}\right)^{\text {inf }}}\right)^{b_{\imath}} \leq \prod_{\imath=1}^{n}\left(\frac{2-\underline{\eta}_{l}}{\underline{\eta}_{\imath}}\right)^{b_{\imath}} \leq \sum_{\imath=1}^{n}\left(\frac{2-\left(\underline{\eta}_{\imath}\right)^{\text {sup }}}{\left(\underline{\eta}_{\imath}\right)^{\text {sup }}}\right)^{b_{\imath}}
$$

if and only if

$$
\begin{aligned}
\left(\frac{2-\left(\underline{\eta}_{\imath}\right)^{\text {inf }}}{\left(\underline{\eta}_{\imath}\right)^{\text {inf }}}\right)^{b_{\imath}} & \leq \prod_{\imath=1}^{n}\left(\frac{2-\underline{\eta}_{\imath}}{\underline{\eta}_{\imath}}\right)^{b_{\imath}} \leq\left(\frac{2-\left(\underline{\eta}_{\imath}\right)^{\text {sup }}}{\left(\underline{\eta}_{\imath}\right)^{\text {sup }}}\right)^{b_{\imath}} \\
& \Longleftrightarrow\left(\frac{2}{\left(\underline{\eta}_{\imath}\right)^{\inf }}\right) \leq \prod_{\imath=1}^{n}\left(\frac{2-\underline{\eta}_{\imath}}{\underline{\eta}_{\imath}}\right)^{b_{\imath}}+1 \leq\left(\frac{2}{\left(\underline{\eta}_{\imath}\right)^{\text {sup }}}\right)
\end{aligned}
$$

which implies that

$$
\left(\frac{\left(\underline{\eta}_{\imath}\right)^{\mathrm{sup}}}{2}\right) \leq \frac{1}{\prod_{\imath=1}^{n}\left(\frac{2-\underline{\eta}_{\imath}}{\underline{\underline{\imath}}_{\imath}}\right)^{b_{\imath}}+1} \leq\left(\frac{\left(\underline{\eta}_{\imath}\right)^{\mathrm{inf}}}{2}\right)
$$


implies that

$$
\left(\underline{\eta}_{\imath}\right)^{\sup } \leq \frac{2}{\prod_{\imath=1}^{n}\left(\frac{2-\underline{\eta}_{\imath}}{\underline{\eta}_{\imath}}\right)^{b_{\imath}}+1} \leq\left(\underline{\eta}_{\imath}\right)^{\inf }
$$

i.e., implies that

$$
\left(\underline{\eta}_{\imath}\right)^{\sup } \leq \frac{2 \prod_{\imath=1}^{n}\left(\underline{\eta}_{\imath}\right)^{b_{\imath}}}{\prod_{\imath=1}^{n}\left(2-\underline{\eta}_{\imath}\right)^{b_{\imath}}+\prod_{\imath=1}^{n}\left(\underline{\eta}_{\imath}\right)^{b_{\imath}}} \leq\left(\underline{\eta}_{\imath}\right)^{\mathrm{inf}}
$$

it also hold even if $\left(\underline{\eta}_{\imath}\right)^{\text {sup }}=0$.

Similarly we can show that

$$
\left(\bar{\nu}_{\imath}\right)^{\mathrm{inf}} \leq \frac{\prod_{\imath=1}^{n}\left(1+\bar{\nu}_{\imath}\right)^{b_{\imath}}-\prod_{\imath=1}^{n}\left(1-\bar{\nu}_{\imath}\right)^{b_{\imath}}}{\prod_{\imath=1}^{n}\left(1+\bar{\nu}_{\imath}\right)^{b_{\imath}}+\prod_{\imath=1}^{n}\left(1-\bar{\nu}_{\imath}\right)^{b_{\imath}}} \leq\left(\bar{\nu}_{\imath}\right)^{\mathrm{sup}}
$$

and

$$
\left(\bar{\eta}_{\imath}\right)^{\sup } \leq \frac{2 \prod_{\imath=1}^{n}\left(\bar{\eta}_{\imath}\right)^{b_{\imath}}}{\prod_{\imath=1}^{n}\left(2-\bar{\eta}_{\imath}\right)^{b_{\imath}}+\prod_{\imath=1}^{n}\left(\bar{\eta}_{\imath}\right)^{b_{\imath}}} \leq\left(\left(\bar{\eta}_{\imath}\right)^{\text {inf }}\right) .
$$

So from above equation we shows that

$$
(\Upsilon(£))^{\mathrm{inf}} \leq I F R E W A\left(\Upsilon\left(£_{1}\right), \Upsilon\left(£_{2}\right), \ldots, \Upsilon\left(£_{n}\right)\right) \leq(\Upsilon(£))^{\mathrm{sup}}
$$

(iii) Monotonicity: Let we have $C\left(L_{\imath}\right)=\left(\underline{C}\left(L_{\imath}\right), \bar{C}\left(L_{\imath}\right)\right)=\left(\underline{g}_{\imath}, \underline{h}_{\imath}\right),\left(\bar{g}_{\imath}, \bar{h}_{\imath}\right)$ and for all $(\imath=1,2, \ldots n) \Upsilon\left(£_{\imath}\right)=\left(\underline{\Upsilon}\left(£_{\imath}\right), \bar{\Upsilon}\left(£_{\imath}\right)\right)=\left(\left(\underline{\nu}_{\imath}, \underline{\eta}_{\imath}\right),\left(\bar{\nu}_{\imath}, \bar{\eta}_{\imath}\right)\right)$ be the collection of IFRVs such that $\underline{C}\left(L_{\imath}\right) \leq \underline{\Upsilon}\left(£_{\imath}\right)$ and $\bar{C}\left(L_{\imath}\right) \leq \bar{\Upsilon}\left(£_{\imath}\right)$. Then to show this

$$
\operatorname{IFREWA}\left(C\left(L_{1}\right), C\left(L_{2}\right), \ldots, C\left(L_{n}\right)\right) \leq \operatorname{IFREWA}\left(\Upsilon\left(£_{1}\right), \Upsilon\left(£_{2}\right), \ldots, \Upsilon\left(£_{n}\right)\right)
$$

Let we have a function $f(c)=\frac{1-c}{1+c}, r \in[0,1]$; the function is decreasing $\underline{g}_{\imath} \leq \underline{\nu}_{\imath}, \forall \imath$, then $f\left(\underline{\nu}_{\imath}\right) \leq f\left(\underline{g}_{\imath}\right) \forall(\imath=1,2, \ldots n)$, i.e. $\frac{1-\underline{\nu}_{\imath}}{1+\underline{\nu}_{\imath}} \leq \frac{1-\underline{g}_{\imath}}{1+\underline{g}_{\imath}},(\imath=1,2, \ldots, n)$. As we know $b=\left(b_{1}, b_{2}, \ldots, b_{n}\right)^{T}$ be weighted vector of $\underline{\Upsilon}\left(£_{\imath}\right)(\imath=1,2, \ldots, n)$ with condition $\sum_{\imath=1}^{n}$ $b_{\imath}=1$ and $b_{\imath} \in[0,1]$. We have

$$
\left(\frac{1-\underline{\nu}_{\imath}}{1+\underline{\nu}_{\imath}}\right)^{b_{\imath}} \leq\left(\frac{1-\underline{g}_{l}}{1+\underline{g}_{\imath}}\right)^{b_{\imath}},(\imath=1,2, \ldots, n) .
$$


Thus

$$
\begin{aligned}
\prod_{\imath=1}^{n}\left(\frac{1-\underline{\nu}_{\imath}}{1+\underline{\nu}_{\imath}}\right)^{b_{\imath}} & \leq \prod_{\imath=1}^{n}\left(\frac{1-\underline{g}_{\imath}}{1+\underline{g}_{\imath}}\right)^{b_{\imath}} \\
& \Longleftrightarrow 1+\prod_{\imath=1}^{n}\left(\frac{1-\underline{\nu}_{\imath}}{1+\underline{\nu}_{\imath}}\right)^{b_{\imath}} \leq 1+\prod_{\imath=1}^{n}\left(\frac{1-\underline{g}_{\imath}}{1+\underline{g}_{\imath}}\right)^{b_{\imath}}
\end{aligned}
$$

if and only if

$$
\begin{aligned}
\frac{1}{1+\prod_{\imath=1}^{n}\left(\frac{1-\underline{g}_{\imath}}{1+\underline{g}_{\imath}}\right)^{b_{\imath}}} & \leq \frac{1}{1+\prod_{\imath=1}^{n}\left(\frac{1-\underline{\nu}_{\imath}}{1+\underline{\nu}_{\imath}}\right)^{b_{\imath}}} \\
& \Longleftrightarrow \frac{2}{1+\prod_{\imath=1}^{n}\left(\frac{1-\underline{g}_{\imath}}{1+\underline{g}_{\imath}}\right)^{b_{\imath}}} \leq \frac{2}{1+\prod_{\imath=1}^{n}\left(\frac{1-\underline{\nu}_{\imath}}{1+\underline{\nu}_{\imath}}\right)^{b_{\imath}}}
\end{aligned}
$$

if and only if

$$
\frac{2}{1+\prod_{\imath=1}^{n}\left(\frac{1-\underline{g}_{\imath}}{1+\underline{g}_{\imath}}\right)^{b_{\imath}}}-1 \leq \frac{2}{1+\prod_{\imath=1}^{n}\left(\frac{1-\underline{\nu}_{\imath}}{1+\underline{\nu}_{\imath}}\right)^{b_{\imath}}}-1
$$

that is

$$
\frac{\prod_{\imath=1}^{n}\left(1+\underline{g}_{\imath}\right)^{b_{\imath}}-\prod_{\imath=1}^{n}\left(1-\underline{g}_{\imath}\right)^{b_{\imath}}}{\prod_{\imath=1}^{n}\left(1+\underline{g}_{\imath}\right)^{b_{\imath}}+\prod_{\imath=1}^{n}\left(1-\underline{g}_{\imath}\right)^{b_{\imath}}} \leq \frac{\prod_{\imath=1}^{n}\left(1+\underline{\nu}_{\imath}\right)^{b_{\imath}}-\prod_{\imath=1}^{n}\left(1-\underline{\nu}_{\imath}\right)^{b_{\imath}}}{\prod_{\imath=1}^{n}\left(1+\underline{\nu}_{\imath}\right)^{b_{\imath}}+\prod_{\imath=1}^{n}\left(1-\underline{\nu}_{\imath}\right)^{b_{\imath}}} .
$$

Now let we have another function $f(y)=\frac{2-y}{y}, y \in(0,1]$, which is decreasing function on $(0,1]$. Since $\left(\underline{h}_{\imath}\right) \geq\left(\underline{\eta}_{\imath}\right)>0, \forall \imath$, then $f\left(\underline{\eta}_{\imath}\right) \geq f\left(\underline{h}_{\imath}\right)$ i.e, $\frac{2-\underline{\eta}_{\imath}}{\underline{\eta}_{\imath}} \geq \frac{2-\underline{h}_{\imath}}{\underline{h}_{\imath}},(\imath=$ $1,2, \ldots, n)$. Let $b=\left(b_{1}, b_{2}, \ldots, b_{n}\right)^{T}$ be weighted vector of $\underline{\Upsilon}\left(£_{\imath}\right)(\imath=1,2, \ldots, n)$ with condition $\sum_{\imath=1}^{n} b_{\imath}=1$ and $b_{\imath} \in[0,1]$. We have

$$
\left(\frac{2-\underline{\eta}_{\imath}}{\underline{\eta}_{\imath}}\right)^{b_{\imath}} \geq\left(\frac{2-\underline{h}_{\imath}}{\underline{h}_{\imath}}\right)^{b_{\imath}},(\imath=1,2, \ldots, n) \text {. }
$$

Thus

if and only if

$$
\prod_{\imath=1}^{n}\left(\frac{2-\underline{\eta}_{\imath}}{\underline{\eta}_{\imath}}\right)^{b_{\imath}} \geq \prod_{\imath=1}^{n}\left(\frac{2-\underline{h}_{\imath}}{\underline{h}_{\imath}}\right)^{b_{\imath}}
$$

if and only if

$$
\prod_{\imath=1}^{n}\left(\frac{2-\underline{\eta}_{\imath}}{\underline{\eta}_{\imath}}\right)^{b_{\imath}}+1 \geq \prod_{\imath=1}^{n}\left(\frac{2-\underline{h}_{\imath}}{\underline{h}_{\imath}}\right)^{b_{\imath}}+1
$$

$$
\frac{1}{\prod_{\imath=1}^{n}\left(\frac{2-\underline{h}_{\imath}}{\underline{h}_{\imath}}\right)^{b_{\imath}}+1} \geq \frac{1}{\prod_{\imath=1}^{n}\left(\frac{2-\underline{\eta}_{\imath}}{\underline{\eta}_{\imath}}\right)^{b_{\imath}}+1}
$$


if and only if

$$
\frac{2}{\prod_{\imath=1}^{n}\left(\frac{2-\underline{h}_{\imath}}{\underline{h}_{\imath}}\right)^{b_{\imath}}+1} \geq \frac{2}{\prod_{\imath=1}^{n}\left(\frac{2-\underline{\eta}_{\imath}}{\underline{\underline{n}}_{\imath}}\right)^{b_{\imath}}+1}
$$

i.e.

$$
\frac{2 \prod_{\imath=1}^{n}\left(\underline{h}_{\imath}\right)^{b_{\imath}}}{\prod_{\imath=1}^{n}\left(2-\underline{h}_{\imath}\right)^{b_{\imath}}+\prod_{\imath=1}^{n}\left(\underline{h}_{\imath}\right)^{b_{\imath}}} \geq \frac{2 \prod_{\imath=1}^{n}\left(\underline{\eta}_{\imath}\right)^{b_{\imath}}}{\prod_{\imath=1}^{n}\left(2-\underline{\eta}_{\imath}\right)^{b_{\imath}}+\prod_{\imath=1}^{n}\left(\underline{\eta}_{\imath}\right)^{b_{\imath}}}
$$

it also hold even if $\underline{\eta}_{\imath}=\underline{h}_{\imath}=0, \forall \imath$,

Similarly we can find

$$
\begin{gathered}
\frac{\prod_{\imath=1}^{n}\left(1+\bar{g}_{\imath}\right)^{b_{\imath}}-\prod_{\imath=1}^{n}\left(1-\bar{g}_{\imath}\right)^{b_{\imath}}}{\prod_{\imath=1}^{n}\left(1+\bar{g}_{\imath}\right)^{b_{\imath}}+\prod_{\imath=1}^{n}\left(1-\bar{g}_{\imath}\right)^{b_{\imath}}} \leq \frac{\prod_{\imath=1}^{n}\left(1+\bar{\nu}_{\imath}\right)^{b_{\imath}}-\prod_{\imath=1}^{n}\left(1-\bar{\nu}_{\imath}\right)^{b_{\imath}}}{\prod_{\imath=1}^{n}\left(1+\bar{\nu}_{\imath}\right)^{b_{\imath}}+\prod_{\imath=1}^{n}\left(1-\bar{\nu}_{\imath}\right)^{b_{\imath}}}, \\
\frac{2 \prod_{\imath=1}^{n}\left(\bar{h}_{\imath}\right)^{b_{\imath}}}{\prod_{\imath=1}^{n}\left(2-\bar{h}_{\imath}\right)^{b_{\imath}}+\prod_{\imath=1}^{n}\left(\bar{h}_{\imath}\right)^{b_{\imath}}} \geq \frac{2 \prod_{\imath=1}^{n}\left(\bar{\eta}_{\imath}\right)^{b_{\imath}}}{\prod_{\imath=1}^{n}\left(2-\bar{\eta}_{\imath}\right)^{b_{\imath}}+\prod_{\imath=1}^{n}\left(\bar{\eta}_{\imath}\right)^{b_{\imath}}} .
\end{gathered}
$$

\subsection{INTUITIONISTIC FUZZY ROUGH EINSTEIN ORDERED WEIGHTED}

AVERAGING OPERATOR. The definition of the IFREOWA operator is introduced in this subsection, and also discussed their fundamental properties.

Definition 10. Suppose the collection $\Upsilon\left(£_{\imath}\right)=\left(\underline{\Upsilon}\left(£_{\imath}\right), \bar{\Upsilon}\left(£_{\imath}\right)\right)=(\imath=1,2,3, \ldots, n)$ of IFRVs having weight vector $b=\left(b_{1}, b_{2}, \ldots, b_{n}\right)^{T}$ satisfying the condition $\sum_{\imath=1}^{n} b_{\imath}=$ 1 and $0 \leq b \leq 1$. Then the IFREOWA operator is defined as follows:

$$
\operatorname{IFREOWA}\left(\Upsilon\left(£_{1}\right), \Upsilon\left(£_{2}\right), \ldots, \Upsilon\left(£_{n}\right)\right)=\left(\oplus_{\imath=1}^{n} b_{\imath} \underline{\Upsilon_{\delta}}\left(£_{\imath}\right), \oplus_{\imath=1}^{n} b_{\imath} \overline{\Upsilon_{\delta}}\left(£_{\imath}\right)\right) \text {. }
$$

Based on this definition the aggregated result for IFREOWA operator is iterated in Theorem 3.

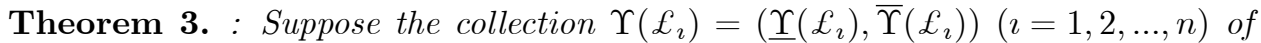
IFRVs having weights vectors $b=\left(b_{1}, b_{2}, \ldots, b_{n}\right)^{T}$ with condition $\sum_{\imath=1}^{n} b_{\imath}=1$ and $0 \leq b_{\imath} \leq 1$. The IFREOWA operator is defined as follows:

$$
\left.\operatorname{IFREOWA}\left(\Upsilon\left(£_{1}\right), \Upsilon\left(£_{2}\right), \ldots, \Upsilon\left(£_{n}\right)\right)\right)=\left(\oplus_{\imath=1}^{n} b_{\imath} \underline{\Upsilon_{\delta}}\left(£_{\imath}\right), \oplus_{\imath=1}^{n} b_{\imath} \overline{\Upsilon_{\delta}}\left(£_{\imath}\right)\right)
$$




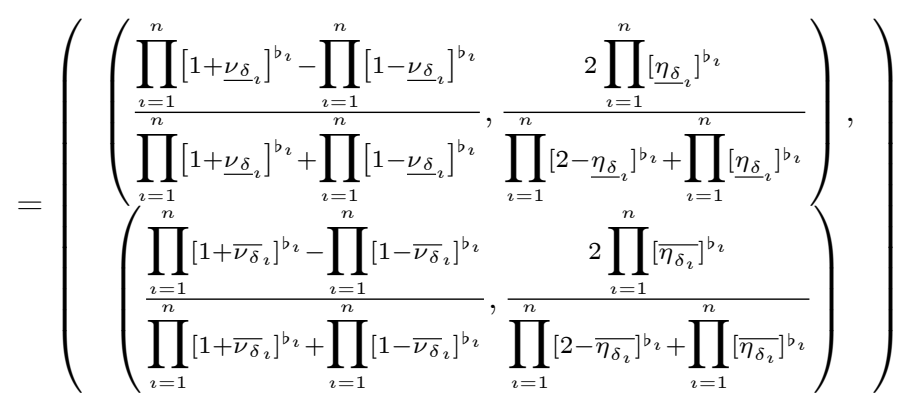

where $\Upsilon_{\delta}\left(£_{\imath}\right)=\left(\Upsilon_{\delta}\left(£_{\imath}\right), \bar{\Upsilon}_{\delta}\left(£_{\imath}\right)\right.$ represent the permutation with the highest value from the set of IFRVs.

Proof. Follow Theorem 1.

Theorem 4. Let us we have a collection $\Upsilon\left(£_{\imath}\right)=\left(\left(\underline{\nu}_{\imath}, \underline{\eta}_{\imath}\right),\left(\bar{\nu}_{\imath}, \bar{\eta}_{\imath}\right)\right)(\imath=1,2, . ., n)$ of IFRVs having weight vectors $b=\left(b_{1}, b_{2}, \ldots, b_{n}\right)^{T}$ with condition $\sum_{r=1}^{n} b_{\imath}=1$ and $0 \leq b_{\imath} \leq 1$. Then their are some essential properties of IFREOWA operator, which are as follows:

(i). (Idempotency): If $\Upsilon\left(£_{\imath}\right)=C(K) \forall \imath=1,2, . ., n$ and $C(K)=(\underline{C}(K), \bar{C}(K))=$ $((\underline{g}, \underline{h}),(\bar{g}, \bar{h}))$. Then

$$
\operatorname{IFREOWA}\left(\Upsilon\left(£_{1}\right), \Upsilon\left(£_{2}\right), \ldots, \Upsilon\left(£_{n}\right)\right)=C(K)
$$

(ii). (Boundedness): Suppose $(\Upsilon(£))^{-}=(\Upsilon(£))^{\inf }=\left(\inf _{\imath} \Upsilon\left(£_{\imath}\right), \sup _{\imath} \bar{\Upsilon}\left(£_{\imath}\right)\right)$ and

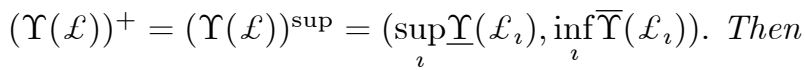

$$
(\Upsilon(£))^{-} \leq I F R E O W A\left(\Upsilon\left(£_{1}\right), \Upsilon\left(£_{2}\right), \ldots, \Upsilon\left(£_{n}\right)\right) \leq(\Upsilon(£))^{+} .
$$

(iii). (Monotonicity): Suppose we have another collection $C\left(L_{\imath}\right)=\left(\underline{C}\left(L_{\imath}\right), \bar{C}\left(L_{\imath}\right)\right)(\imath=$ $1,2, \ldots n)$ of IFRVs such that $\underline{C}\left(L_{\imath}\right) \leq \underline{\Upsilon}\left(£_{\imath}\right)$ and $\bar{C}\left(L_{\imath}\right) \leq \bar{\Upsilon}\left(£_{\imath}\right)$. Then

$$
\text { IFREOW } A\left(C\left(L_{1}\right), C\left(L_{2}\right), \ldots, C\left(L_{n}\right)\right) \leq \operatorname{IFREOWA}\left(\Upsilon\left(£_{1}\right), \Upsilon\left(£_{2}\right), \ldots, \Upsilon\left(£_{n}\right)\right)
$$

Proof. For proof follow Theorem 2.

\subsection{INTUITIONISTIC FUZZY ROUGH EINSTEIN HYBRID AVER-}

AGING OPERATOR. We will introduce the IFREHA operator in this section, which weights both the value and the order of an IF argument at the same time. The initiated operator's essential properties are explored in depth.

Definition 11. Suppose the collection $\Upsilon\left(£_{\imath}\right)=\left(\underline{\Upsilon}\left(£_{\imath}\right), \bar{\Upsilon}\left(£_{\imath}\right)\right)(\imath=1,2,3 \ldots, n)$ of IFRVs having weight vector $b=\left(b_{1}, b_{2}, \ldots, b_{n}\right)^{T}$ with condition $\sum_{\imath=1}^{n} b_{\imath}=1$ and $0 \leq b \leq 1$. Let $\varrho=\left(\varrho_{1}, \varrho_{2}, \ldots, \varrho_{n}\right)^{T}$ be the associated weight vector of the given collection with condition $\sum_{\imath=1}^{n} \varrho_{\imath}=1$ and $0 \leq \varrho \leq 1$. Then IFREHA operator is defined as:

$$
\begin{aligned}
(3.3) \operatorname{IFREH} A\left(\Upsilon\left(£_{1}\right), \Upsilon\left(£_{2}\right), \ldots, \Upsilon\left(£_{n}\right)\right) & =\oplus_{\imath=1}^{n} b_{\imath} \Upsilon^{\prime}\left(£_{\imath}\right) \\
& =\left[\oplus_{\imath=1}^{n} b_{\imath} \underline{\Upsilon_{\delta}^{\prime}}\left(£_{\imath}\right), \oplus_{\imath=1}^{n} b_{\imath} \overline{\Upsilon_{\delta}^{\prime}}\left(£_{\imath}\right)\right]
\end{aligned}
$$

Based on this definition the aggregated result for IFREHA operator is iterated in Theorem 5. 
Theorem 5. suppose we have a collection $\Upsilon\left(£_{\imath}\right)=\left(\underline{\Upsilon}\left(£_{\imath}\right), \bar{\Upsilon}\left(£_{\imath}\right)\right)(\imath=1,2,3 \ldots, n)$ of IFRVs having weight vectors $b=\left(b_{1}, b_{2}, \ldots, b_{n}\right)^{T}$ such that $\sum_{\imath=1}^{n} b_{\imath}=1$ and $0 \leq b \leq 1$. Let $\varrho=\left(\varrho_{1}, \varrho_{2}, \ldots, \varrho_{n}\right)^{T}$ be the associated weight vector of the given collection with condition $\sum_{\imath=1}^{n} \varrho_{\imath}=1$ and $0 \leq \varrho \leq 1$. The IFREHA is then defined as follows:

$$
\begin{aligned}
& \operatorname{IFREHA}\left(\Upsilon\left(£_{1}\right), \Upsilon\left(£_{2}\right), \ldots, \Upsilon\left(£_{n}\right)\right)=\oplus_{\imath=1}^{n} b_{\imath} \Upsilon^{\prime}\left(£_{\imath}\right) \\
& =\left[\oplus_{\imath=1}^{n} b_{\imath} \underline{\Upsilon_{\delta}^{\prime}}\left(£_{\imath}\right), \oplus_{\imath=1}^{n} b_{\imath} \overline{\Upsilon_{\delta}^{\prime}}\left(£_{\imath}\right)\right]
\end{aligned}
$$

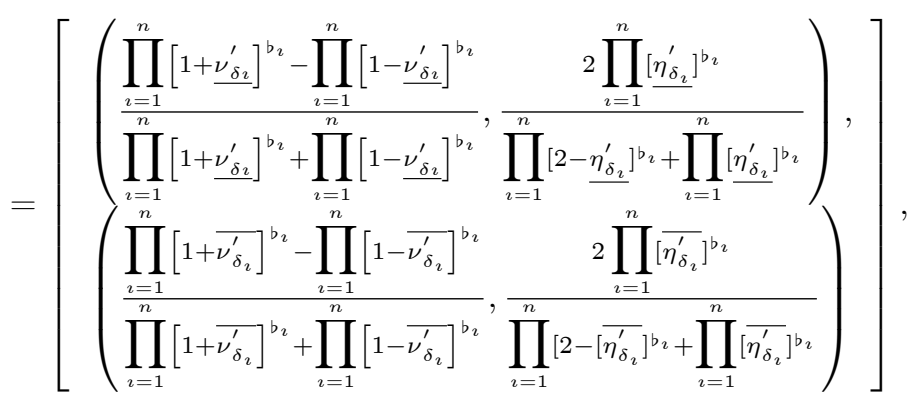

where $\Upsilon^{\prime}\left(£_{\imath}\right)=n b_{\imath} \Upsilon\left(£_{\imath}\right)\left(n b_{\imath} \underline{\Upsilon_{\delta}^{\prime}}\left(£_{\imath}\right), n b_{\imath} \overline{\Upsilon_{\delta}^{\prime}}\left(£_{\imath}\right)\right), n$ is the balancing coefficient, represent the permutation with the highest value from the set of IFRVs.

Proof. Follow Theorem 1.

The given IFREHA operator was reduced to IFREOWA operator if $b=\left(\frac{1}{b_{1}}, \frac{1}{b_{2}}, \ldots, \frac{1}{b_{n}}\right)^{T}$ operator.

Theorem 6. suppose we have a collection $\Upsilon\left(£_{\imath}\right)=\left(\underline{\Upsilon}\left(£_{\imath}\right), \bar{\Upsilon}\left(£_{\imath}\right)\right)(\imath=1,2,3 \ldots, n)$ of IFRVs having weight vectors $b=\left(b_{1}, b_{2}, \ldots, b_{n}\right)^{T}$ such that $\sum_{\imath=1}^{n} b_{\imath}=1$ and $0 \leq b \leq 1$. Let $\varrho=\left(\varrho_{1}, \varrho_{2}, \ldots, \varrho_{n}\right)^{T}$ be the associated weight vector of the given collection with condition $\sum_{\imath=1}^{n} \varrho_{\imath}=1$ and $0 \leq \varrho \leq 1$. Some of the properties of IFREHA operators are as follows:

(i). (Idempotency): If $\Upsilon\left(£_{\imath}\right)=C(K) \forall \imath=1,2, . ., n$ and $C(K)=(\underline{C}(K), \bar{C}(K))=$ $((\underline{g}, \underline{h}),(\bar{g}, \bar{h}))$. Then

$$
\operatorname{IFREHA}\left(\Upsilon\left(£_{1}\right), \Upsilon\left(£_{2}\right), \ldots, \Upsilon\left(£_{n}\right)\right)=C(K)
$$

(ii). (Boundedness): Suppose $(\Upsilon(£))^{-}=(\Upsilon(£))^{\inf }=\left(\inf _{\imath} \Upsilon\left(£_{\imath}\right), \sup \bar{\Upsilon}\left(£_{\imath}\right)\right)$ and $(\Upsilon(£))^{+}=(\Upsilon(£))^{\sup }=\left(\sup _{\imath} \Upsilon\left(£_{\imath}\right), \inf _{\imath} \bar{\Upsilon}\left(£_{\imath}\right)\right)$. Then

$$
(\Upsilon(£))^{-} \leq \operatorname{IFREHA}\left(\Upsilon\left(£_{1}\right), \Upsilon\left(£_{2}\right), \ldots, \Upsilon\left(£_{n}\right)\right) \leq(\Upsilon(£))^{+} .
$$

(iii). (Monotonicity): Suppose we have another collection $C\left(L_{\imath}\right)=\left(\underline{C}\left(L_{\imath}\right), \bar{C}\left(L_{\imath}\right)\right)(\imath=$ $1,2, \ldots n)$ of IFRVs such that $\underline{C}\left(L_{\imath}\right) \leq \underline{\Upsilon}\left(£_{\imath}\right)$ and $\bar{C}\left(L_{\imath}\right) \leq \bar{\Upsilon}\left(£_{\imath}\right)$.

$$
\operatorname{IFREHA}\left(C\left(L_{1}\right), C\left(L_{2}\right), \ldots, C\left(L_{n}\right)\right) \leq \operatorname{IFREHA}\left(\Upsilon\left(£_{1}\right), \Upsilon\left(£_{2}\right), \ldots, \Upsilon\left(£_{n}\right)\right)
$$

Proof. Follow from Theorem 2. 


\section{Methodological Development of Intuitionistic Fuzzy Rough Entropy Measure}

Based on the distance model $[54,55]$, this part presented the generalized distance and weighted generalized distance measures using intuitionistic fuzzy rough information to determine the differences between the two IFRSs, After that, we presented a novel entropy measure for IFRSs to measure its fuzziness, based on generalized distance measures.

\subsection{IFRSs Distance Measure.}

Definition 12. Let

$$
\Upsilon(£)=\left\{\Upsilon\left(£_{1}\right), \Upsilon\left(£_{2}\right), \ldots, \Upsilon\left(£_{n}\right)\right\}
$$

and

$$
\Upsilon^{*}(£)=\left\{\Upsilon^{*}\left(£_{1}\right), \Upsilon^{*}\left(£_{2}\right), \ldots, \Upsilon^{*}\left(£_{n}\right)\right\}
$$

be two IFRSs, where $\Upsilon\left(£_{\imath}\right)=\left(\left(\underline{\Upsilon}\left(£_{\imath}\right), \bar{\Upsilon}\left(£_{\imath}\right)\right)\right)=\left(\left(\underline{\nu}_{\imath}, \underline{\eta}_{\imath}\right),\left(\bar{\nu}_{\imath}, \bar{\eta}_{\imath}\right)\right)$ and $\Upsilon^{*}\left(£_{\imath}\right)=$ $\left(\left(\underline{\Upsilon}^{*}\left(£_{\imath}\right), \bar{\Upsilon}^{*}\left(£_{\imath}\right)\right)\right)=\left(\left(\underline{\nu}_{\imath}^{*}, \underline{\eta}_{\imath}^{*}\right),\left(\bar{\nu}_{\imath}^{*}, \bar{\eta}_{\imath}^{*}\right)\right) \imath=\{1,2,3, \ldots, n\}$. Then for any $\lambda>$ $0(\in \mathbb{R})$ the generalized distance measure between any two IFRSs are defined as follows:

$$
d_{G D}\left(\Upsilon(£), \Upsilon^{*}(£)\right)=\left(\frac{1}{4 n} \sum_{\imath=1}^{n}\left[\begin{array}{c}
\left|\underline{\nu}_{\imath}-\underline{\nu}_{l}^{*}\right|^{\lambda}+\left|\underline{\eta}_{\imath}-\underline{\eta}_{\imath}^{*}\right|^{\lambda}+ \\
\left|\bar{\nu}_{\imath}-\bar{\nu}_{\imath}^{*}\right|^{\lambda}+\left|\bar{\eta}_{\imath}-\bar{\eta}_{\imath}^{*}\right|^{\lambda}
\end{array}\right]\right)^{\frac{1}{\lambda}} .
$$

Definition 13. For any $\lambda \in(\mathbb{R})>0$ the weighted generalized distance measure for two IFRSs

$$
\Upsilon(£)=\left\{\Upsilon\left(£_{1}\right), \Upsilon\left(£_{2}\right), \ldots, \Upsilon\left(£_{n}\right)\right\}
$$

and

$$
\Upsilon^{*}(£)=\left\{\Upsilon^{*}\left(£_{1}\right), \Upsilon^{*}\left(£_{2}\right), \ldots, \Upsilon^{*}\left(£_{n}\right)\right\}
$$

where $\Upsilon\left(£_{\imath}\right)=\left(\left(\underline{\Upsilon}\left(£_{\imath}\right), \bar{\Upsilon}\left(£_{\imath}\right)\right)\right)=\left(\left(\underline{\nu}_{\imath}, \underline{\eta}_{\imath}\right),\left(\bar{\nu}_{\imath}, \bar{\eta}_{\imath}\right)\right)$ and $\Upsilon^{*}\left(£_{\imath}\right)=\left(\left(\underline{\Upsilon}^{*}\left(£_{\imath}\right), \bar{\Upsilon}^{*}\left(£_{\imath}\right)\right)\right)=$ $\left(\left(\underline{\nu}_{\imath}^{*}, \underline{\eta}_{\imath}^{*}\right),\left(\bar{\nu}_{\imath}^{*}, \bar{\eta}_{\imath}^{*}\right)\right) \imath=\{1,2,3, \ldots, n\}$ are defined as under.

$$
d_{W G D}\left(\Upsilon(£), \Upsilon^{*}(£)\right)=\left(\frac{1}{4 n} \sum_{\imath=1}^{n} b_{\imath}\left[\begin{array}{c}
\left|\underline{\nu}_{\imath}-\underline{\nu}_{\imath}^{*}\right|^{\lambda}+\left|\underline{\eta}_{\imath}-\underline{\eta}_{\imath}^{*}\right|^{\lambda}+ \\
\left|\bar{\nu}_{\imath}-\bar{\nu}_{\imath}^{*}\right|^{\lambda}+\left|\bar{\eta}_{\imath}-\bar{\eta}_{\imath}^{*}\right|^{\lambda}
\end{array}\right]\right)^{\frac{1}{\lambda}} .
$$

where $b_{\imath}(\imath=1,2, \ldots, n)$ denotes the weights such that $b_{\imath} \geq 0$ and $\sum_{j=1}^{n} b_{\imath}=1$.

Remark 1. (1) The above mentioned distance measure are said to be Hamming distance, if $\lambda=1$.

(2) The above mentioned distance measure are said to be Euclidean distance, if $\lambda=2$.

Definition 14. Let $\Upsilon(£)=((\underline{\nu}, \underline{\eta}),(\bar{\nu}, \bar{\eta}))$ and $\Upsilon^{*}(£)=\left(\left(\underline{\nu}^{*}, \underline{\eta}^{*}\right),\left(\bar{\nu}^{*}, \bar{\eta}^{*}\right)\right)$ be two IFRSs. Then the equation (13) definition of Generalized distance measure (GDM) can be expressed as follow:

$$
d_{G D}\left(\Upsilon(£), \Upsilon^{*}(£)\right)=\left(\frac{1}{4}\left[\begin{array}{c}
\left|\underline{\nu}-\underline{\nu}^{*}\right|^{\lambda}+\left|\underline{\eta}-\underline{\eta}^{*}\right|^{\lambda}+ \\
\left|\bar{\nu}-\bar{\nu}^{*}\right|^{\lambda}+\left|\bar{\eta}-\bar{\eta}^{*}\right|^{\lambda}
\end{array}\right]\right)^{\frac{1}{\lambda}} \lambda>0(\in \mathbb{R}) .
$$


For any two $\Upsilon(£), \Upsilon^{*}(£) \in I F R S\left(p^{*}\right)$, satisfying the following conditions.

(1) $d\left(\Upsilon(£), \Upsilon^{*}(£)\right) \in[0,1]$,

(2) $d\left(\Upsilon(£), \Upsilon^{*}(£)\right)=1$, iff $\Upsilon(£)=\Upsilon^{*}(£)$,

(3) $d\left(\Upsilon(£), \Upsilon^{*}(£)\right)=d\left(\Upsilon^{*}(£), \Upsilon(£)\right)$.

4.2. Entropy Measure for IFRSs. On the basis of Guo and Song's definition [54], This part proposes a new entropy measure for IFRSs.

Definition 15. Let $\Upsilon(£)=\left\{\Upsilon\left(£_{1}\right), \Upsilon\left(£_{2}\right), \ldots, \Upsilon\left(£_{n}\right)\right\}$ be the IFRSs, then for each $\imath=\{1,2,3, \ldots, n\}$ where $\Upsilon\left(£_{\imath}\right)=\left(\underline{\Upsilon}\left(£_{\imath}\right), \bar{\Upsilon}\left(£_{\imath}\right)\right)=\left(\left(\underline{\nu}_{\imath}, \underline{\eta}_{\imath}\right),\left(\bar{\nu}_{\imath}, \bar{\eta}_{\imath}\right)\right)$. Then entropy measure for IFRV $\Upsilon(£)$ can be expressed as follows:

$$
E(\Upsilon(£))=\frac{1}{n} \sum_{\imath=1}^{n}\left[\left\{1-d\left(\Upsilon\left(£_{\imath}\right), \Upsilon^{c}\left(£_{\imath}\right)\right)\right\}\left(\frac{1+\Xi \underline{\Upsilon}\left(£_{\imath}\right)+\Xi \bar{\Upsilon}\left(£_{\imath}\right)}{3}\right)\right] .
$$

\section{IFRS EXTENDED TOPSIS}

5.1. Intuitionistic fuzzy rough MCGDM problem. In terms of intuitionistic fuzzy rough informations, we suggest an approach for solving MCGDM problems. A decision matrix can be used to express MCGDM, where the attributes (criteria) are expressed by columns, and alternatives are expressed by row. Suppose we have a set which consists $m$ number of alternatives represented by $\Upsilon=$ $\left\{p_{1}^{*}, p_{2}^{*}, \ldots, p_{m}^{*}\right\}$ and a set consists $n$ number of attributes (criteria) represented as $C=\left\{c_{1}^{*}, c_{2}^{*}, \ldots, c_{n}^{*}\right\}$. Also, suppose there are set consists $e$ number of DMs, denoted by $\check{D}=\left\{\check{D}_{1}, \check{D}_{2}, \ldots, \check{D}_{e}\right\}$, who provided their assessment report for each alternative $\Upsilon_{i}(i=1,2,3, \ldots, m)$ against their attributes (criteria) $C_{j}(j=1,2,3, \ldots, n)$. Let $b=\left(b c_{1}, b c_{2}, \ldots, b c_{n}\right)^{T}$ represent the unknown weight vector for attributes (criteria) with $\sum_{j=1}^{n} b c_{j}=1$ and $b c_{j} \in[0,1]$.The intuitionistic fuzzy rough decision matrix is assumed to be denoted by $\Theta^{l}=\left[\Upsilon\left(£_{i j}^{l}\right)\right]_{m \times n}=\left\langle\left(\underline{\nu}_{i j}^{(l)}, \underline{\eta}_{i j}^{(l)}\right),\left(\bar{\nu}_{i j}^{(l)}, \bar{\eta}_{i j}^{(l)}\right)\right\rangle_{m \times n}$, $l \in 1,2, \ldots, e$, where as $\underline{\nu}_{i j}, \underline{\eta}_{i j}$ is the degree of lower membership and non membership and $\bar{\nu}_{i j}, \bar{\eta}_{i j}$ is the upper member and non membership assumed by decision makers (DMs),

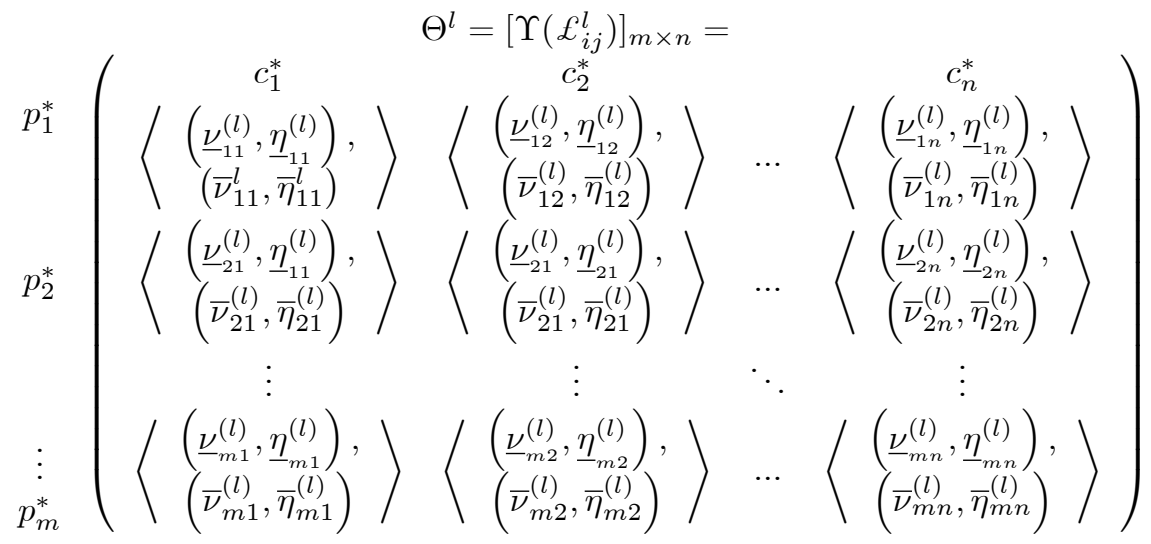

It should be noted that in the decision-making process, all details about the weights of (DM's) and attributes (criteria) is completely unknown. 


\subsection{INTUITIONISTIC FUZZY ROUGH EXTENDED TOPSIS METHOD.}

The method is divided into five parts. In the first part, a TOPSIS-based method for computing decision-makers (DMs) weights is proposed. Second part consists of computing the weights of attributes (criteria) with help of new proposed entropy measure. With PIS and NIS, the final aspect is a rating system based on degree of similarity to the ideal solution.

The following steps are developed for solving MCGDM problem with IFRVs using a TOPSIS-based procedure:

Step-(1): Normalize the decision matrix $\Theta^{l}=\left[\Upsilon\left(£\left({ }_{i j}^{l)}\right)\right]_{m \times n}\right.$. In an MCGDM problem, the attributes/criteria have two different types. They're profit and cost criteria, and their principles have opposing effects on decision-making. The values of cost criteria in $\Theta^{l}=\left[\Upsilon\left(£\left(\begin{array}{l}l) \\ i j\end{array}\right)\right]_{m \times n}\right.$ must be normalized to unify such results, and a normalized matrix is obtained as follows:

$$
N_{i j}^{*(l)}= \begin{cases}\left(\underline{\nu}_{i j}, \underline{\eta}_{i j}\right),\left(\bar{\nu}_{i j}, \bar{\eta}_{i j}\right) & \text { for profit criteria } \\ \left(\underline{\eta}_{i j}, \underline{\nu}_{i j}\right),\left(\bar{\eta}_{i j}, \bar{\nu}_{i j}\right) & \text { for cost criteria }\end{cases}
$$

Step-(2a): It is difficult to achieve the final decision outcome if the DM's weight are undefined. Initially the weights of DMs' are computed as follows: Since the group decision $\left(G^{*} D \tilde{I} S^{\prime}\right)$ is nearer to all of the opinions of each individual DM's. Consequently, the better $\left(G^{*} D \tilde{I} S^{\prime}\right)$ can be calculated by averaging all of the opinions of each individual DM's. We calculate the $\left(G^{*} D \tilde{I} S^{\prime}\right)$ in this step by taking intuitionistic fuzzy rough Einstein weighted average of alternatives value corresponding to the criteria provided by the DM's, while DM's values are given the same weighted:

$$
G^{*} D \tilde{I} \dot{S}=\left(\begin{array}{cccc}
\tilde{I} \dot{S}_{11} & \tilde{I} \dot{S}_{12} & \ldots & \tilde{I} \dot{S}_{1 n} \\
\tilde{I} \dot{S}_{21} & \tilde{I} \dot{S}_{22} & \ldots & \tilde{I} \dot{S}_{2 n} \\
\vdots & \vdots & \ddots & \vdots \\
\tilde{I} \dot{S}_{m 1} & \tilde{I} \dot{S}_{m 2} & \ldots & \tilde{I} \dot{S}_{m n}
\end{array}\right)
$$

Where

$$
\begin{aligned}
& \breve{I} \hat{S}_{i j}=\sum_{l=1}^{e} \frac{1}{e} N_{i j}^{*(l)}
\end{aligned}
$$

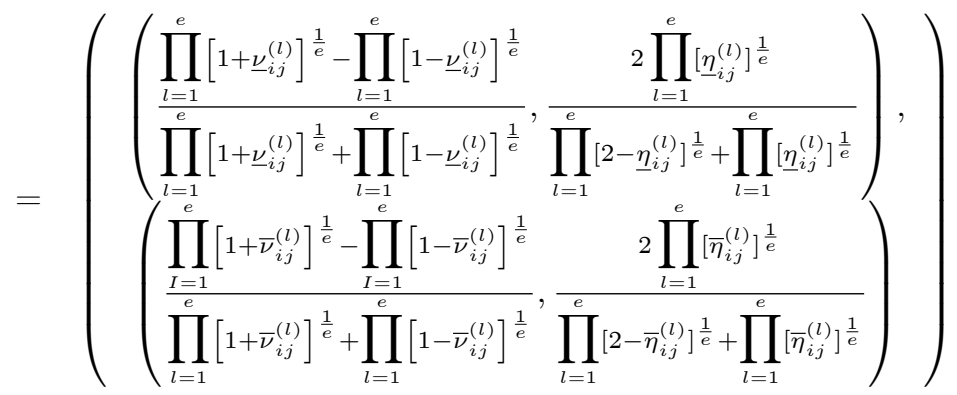


Step-(2b): The group left ideal decision $\left(G^{*} \dot{L} \tilde{I} D\right)$ and group right ideal decision $\left(G^{*} \dot{R} \tilde{I} D\right)$ are determined as follows:

$$
G^{*} \dot{R} \tilde{I} D=\left(\begin{array}{cccc}
\dot{R} \tilde{I} D_{11} & \dot{R} \tilde{I} D_{12} & \ldots & \dot{R} \tilde{I} D_{1 n} \\
\dot{R} \tilde{I} D_{21} & \dot{R} \tilde{I} D_{22} & \ldots & \dot{R} \tilde{I} D_{21} \\
\vdots & \vdots & \ddots & \vdots \\
\dot{R} \tilde{I} D_{m 1} & \dot{R} \tilde{I} D_{m 1} & \ldots & \dot{R} \tilde{I} D_{m n}
\end{array}\right)
$$

Where

$$
\dot{R} \tilde{I} D_{i j}=\left\{\left(N_{i j}^{*(l)}\right): \max _{l}\left[\left(\mathrm{~S}\left(N_{i j}^{*(l)}\right)\right]\right\}\right.
$$

and

$$
G^{*} \dot{L} \tilde{I} D=\left(\begin{array}{cccc}
\dot{L} \tilde{I} D_{11} & \dot{L} \tilde{I} D_{12} & \ldots & \dot{L} \tilde{I} D_{1 n} \\
\dot{L} \tilde{I} D_{21} & \dot{L} \tilde{I} D_{22} & \ldots & \dot{L} \tilde{I} D_{21} \\
\vdots & \vdots & \ddots & \vdots \\
\dot{L} \tilde{I} D_{m 1} & \dot{L} \tilde{I} D_{m 1} & \ldots & \dot{L} \tilde{I} D_{m n}
\end{array}\right)
$$

Where

$$
\dot{L} \tilde{I} D_{i j}=\left\{\left(N_{i j}^{*(l)}\right): \min _{l}\left[\subseteq\left(N_{i j}^{*(l)}\right)\right]\right\} .
$$

for $(i=1,2, \ldots m)$ and $(j=1,2, \ldots n)$.

Step-(2c): Here, we use equation (4.1) to compute the distance of decision matrices $N_{i j}^{*(l)}$ to $G^{*} D \breve{I} \hat{S}, G^{*} \tilde{R} \tilde{I} D$ and $G^{*} \tilde{L} \tilde{I} D$, and symbolically represented by $D G^{*} D \tilde{I} \tilde{S}, D G^{*} \hat{R} \tilde{I} D$ and $D G^{*} \tilde{L} \tilde{I} D$ respectively, which are mentioned below:

$D G^{*} D \tilde{I} \dot{S}_{i}^{(l)}=\left(\frac{1}{4 n} \sum_{j=1}^{n}\left(\begin{array}{c}\left|\underline{\nu}_{N_{i j}^{*(l)}}-\underline{\nu}_{\tilde{I} \dot{S}_{i j}}\right|^{\lambda}+\left|\underline{\eta}_{N_{i j}^{*(l)}}-\underline{\eta}_{\tilde{I} \hat{S}_{i j}}\right|^{\lambda} \\ +\left|\bar{\nu}_{N_{i j}^{*(l)}}-\bar{\nu}_{\tilde{I} \dot{S}_{i j}}\right|^{\lambda}+\left|\bar{\eta}_{N_{i j}^{*(l)}}-\bar{\eta}_{\tilde{I} \dot{S}_{i j}}\right|^{\lambda}\end{array}\right)\right)^{\frac{1}{\lambda}}$,

$D G^{*} \dot{R} \tilde{I} D_{i}^{(l)}=\left(\frac{1}{4 n} \sum_{j=1}^{n}\left(\begin{array}{c}\left|\underline{\nu}_{N_{i j}^{*(l)}}-\underline{\nu}_{\hat{R} \tilde{I} D_{i j}}\right|^{\lambda}+\left|\underline{\eta}_{N_{i j}^{*(l)}}-\underline{\eta}_{\hat{R} \tilde{I} D_{i j}}\right|^{\lambda}+ \\ \left|\bar{\nu}_{N_{i j}^{*(l)}}-\bar{\nu}_{\dot{R} \tilde{I} D_{i j}}\right|^{\lambda}+\left|\bar{\eta}_{N_{i j}^{*(l)}}-\bar{\eta}_{\dot{R} \tilde{I} D_{i j}}\right|^{\lambda}\end{array}\right)\right)^{\frac{1}{\lambda}}$,

$D G^{*} \dot{L} \tilde{I} D_{i}^{(l)}=\left(\frac{1}{4 n} \sum_{j=1}^{n}\left(\begin{array}{c}\left|\underline{\nu}_{N_{i j}^{*(l)}}-\underline{\nu}_{\dot{L} \tilde{I} D_{i j}}\right|^{\lambda}+\left|\underline{\eta}_{N_{i j}^{*(l)}}-\underline{\eta}_{\dot{L} \tilde{I} D_{i j}}\right|^{\lambda}+ \\ \left|\bar{\nu}_{N_{i j}^{*(l)}}-\bar{\nu}_{\dot{L} \tilde{I} D_{i j}}\right|^{\lambda}+\left|\bar{\eta}_{N_{i j}^{*(l)}}-\bar{\eta}_{\dot{L} \tilde{I} D_{i j}}\right|^{\lambda}\end{array}\right)\right)^{\frac{1}{\lambda}}$,

for $i=1,2, \ldots, m$ and $l=1,2, \ldots, e$.

Step-(2d): Following the model proposed [53], We measure the closeness indices (CIs) as follows:

$\dot{C}^{*} I^{(l)}=\frac{\sum_{i=1}^{m} D G^{*} \dot{R} \tilde{I} D_{i}^{(l)}+\sum_{i=1}^{m} D G^{*} \dot{L} \tilde{I} D_{i}^{(l)}}{\sum_{i=1}^{m} D G^{*} D \tilde{I} \tilde{S}_{i}^{(l)}+\sum_{i=1}^{m} D G^{*} \hat{R} \tilde{I} D_{i}^{(l)}+\sum_{i=1}^{m} D G^{*} \dot{L} \tilde{I} D_{i}^{(l)}}$.

For $l=1,2, \ldots, e$. 
Step-(2e): In this step, by using following formula to determined the DMs weights:

$$
b^{(l)}=\frac{\dot{C}^{*} I^{l}}{\sum_{l=1}^{e} \dot{C}^{*} I^{l}}
$$

Step-(3a): Using the proposed IFRVs entropy measure to find the weights of attributes (criteria), and the revised group decision $\left(R G^{*} D \tilde{I} \tilde{S}^{\prime}\right)$ was calculated as follows:

$$
\begin{aligned}
& R_{i j}^{*} \tilde{I} \dot{S}=\sum_{I=1}^{e} b^{(l)} N_{i j}^{*(l)} \\
& =\left(\begin{array}{c}
\left(\frac{\prod_{l=1}^{e}\left[1+\underline{\nu}_{i j}^{(l)}\right]^{b(l)}-\prod_{l=1}^{e}\left[1-\underline{\nu}_{i j}^{(l)}\right]^{b^{(l)}}}{\prod_{l=1}^{e}\left[1+\underline{\nu}_{i j}^{(l)}\right]^{b^{(l)}}+\prod_{l=1}^{e}\left[1-\underline{\underline{\nu}}_{i j}^{(l)}\right]^{b^{(l)}}}, \frac{2 \prod_{l=1}^{e}\left[\underline{\underline{r}}_{i j}^{(l)}\right]^{b^{(l)}}}{\prod_{l=1}^{e}\left[2-\underline{\underline{\eta}}_{i j}^{(l)}\right]^{\mathrm{b}(l)}+\prod_{l=1}^{e}\left[\underline{\eta}_{i j}^{(l)} \mathrm{b}^{(l)}\right.}\right), \\
\left(\frac{\prod_{I=1}^{e}\left[1+\bar{\nu}_{i j}^{(l)}\right]^{b^{(l)}}-\prod_{I=1}^{e}\left[1-\bar{\nu}_{i j}^{(l)}\right]^{(l)}}{\prod_{l=1}^{e}\left[1+\bar{\nu}_{i j}^{(l)}\right]^{b^{(l)}}+\prod_{l=1}^{e}\left[1-\bar{\nu}_{i j}^{(l)}\right]^{(l)}}, \frac{2 \prod_{l=1}^{e}\left[\bar{\eta}_{i j}^{(l)}\right]^{(l)}}{\prod_{l=1}^{e}\left[2-\bar{\eta}_{i j}^{(l)} b^{(l)}+\prod_{l=1}^{e}\left[\bar{\eta}_{i j}^{(l)}\right]^{b(l)}\right.}\right)
\end{array}\right)
\end{aligned}
$$

Step-(3b): The entropy measure for each attribute in IFRV is determined by using Equation (4.4):

$$
E c_{j}=E\left(R^{*} \tilde{I} \dot{S}_{1 j}, R^{*} \tilde{I} \dot{S}_{2 j}, \ldots, R^{*} \tilde{I} \dot{S}_{m j}\right), j=1,2, \ldots, n .
$$

Step-(3c): The following formula is used to measure attribute weights:

$$
b_{c_{j}}=\frac{1-E c_{j}}{n-\sum_{j=1}^{n} E c_{j}}, j=1,2, \ldots, n .
$$

Step-(4a): The weighted normalized decision matrices by using criteria weight vector are determined as follows:

$$
\begin{aligned}
& D M\left(N^{*}\right)_{i j}^{(l)}=\sum_{I=1}^{e} b c_{j} N_{i j}^{*(l)} \\
& =\left(\begin{array}{c}
\left(\frac{\left[1+\underline{\nu}_{i j}^{(l)}\right]^{b_{c_{j}}}-\left[1-\underline{\nu}_{i j}^{(l)}\right]^{b_{c_{j}}}}{\left[1+\underline{\nu}_{i j}^{(l)}\right]^{b_{c_{j}}}+\left[1-\underline{\nu}_{i j}^{(l)}\right]^{b_{c_{j}}}}, \frac{2\left[\underline{\eta}_{i j}^{(l)}\right]^{b_{c_{j}}}}{\left[2-\underline{\eta}_{i j}^{(l)}\right]^{b c_{j}}+\left[\underline{\eta}_{i j}^{(l)}\right]^{b c_{j}}}\right), \\
\left(\frac{\left[1+\bar{\nu}_{i j}^{(l)}\right]^{b_{c_{j}}}-\left[1-\bar{\nu}_{i j}^{(l)}\right]^{b_{c_{j}}}}{\left[1+\bar{\nu}_{i j}^{(l)}\right]^{b_{c_{j}}}+\left[1-\bar{\nu}_{i j}^{(l)}\right]^{b_{c_{j}}}}, \frac{2\left[\bar{\eta}_{i j}^{(l)}\right]^{c_{c_{j}}}}{\left[2-\bar{\eta}_{i j}^{(l)}\right]^{b C_{j}}+\left[\bar{\eta}_{i j}^{(l)}\right]^{b_{c_{j}}}}\right)
\end{array}\right)
\end{aligned}
$$

for each $(l=1,2, \ldots, e)$.

Step-(4b): Determine $P^{*} I S^{(l)}$ and $N^{*} I S^{(l)}$ for each DM's by using weighted normalized decision matrices $D M\left(N^{*}\right)_{i j}^{(l)}$, are defined as follows:

$P^{*} I S^{(l)}=\left\{\left(D M\left(N^{*}\right)_{i j}^{(l)}\right): \max _{i}\left[\left(S\left(D M\left(N^{*}\right)_{i j}^{(l)}\right)\right]\right\}, \quad(j=1,2, \ldots, n)\right.$

and

$N^{*} I S^{(l)}=\left\{\left(D M(N)_{i j}^{(l)}\right): \min _{i}\left[\subseteq\left(D M\left(N^{*}\right)_{i j}^{(l)}\right)\right]\right\}, \quad(j=1,2, \ldots, n)$ 
Step-(4c): By using equation 4.2 the WGDM from $D M\left(N^{*}\right)^{(l)}$ to $P^{*} I S^{(l)}$ and $N^{*} I S^{(l)}$, are computed as follows:

$$
=\left(\frac{1}{4 n} \sum_{j=1}^{n} b c_{j}\left(\begin{array}{c}
\left|\left(\underline{\nu}_{D M\left(N^{*}\right)^{(l)}}\right)-\left(\underline{\nu}_{P^{*} I S^{(l)}}\right)\right|^{\lambda(l)}+\left|\left(\underline{\eta}_{D M\left(N^{*}\right)^{(l)}}\right)-\left(\underline{\eta}_{P^{*} I S^{(l)}}\right)\right|^{\lambda} \\
\left|\left(\bar{\nu}_{D M\left(N^{*}\right)^{(l)}}\right)-\left(\bar{\nu}_{\left.P^{*} I S^{(l)}\right)}\right)\right|^{\lambda}+\left|\left(\bar{\eta}_{D M\left(N^{*}\right)^{(l)}}\right)-\left(\bar{\eta}_{P^{*} I S^{(l)}}\right)\right|^{\lambda}+
\end{array}\right)\right)^{\frac{1}{\lambda}}
$$

and

$$
\begin{aligned}
& D I S_{i}^{-(l)} \\
& =\left(\frac{1}{4 n} \sum_{j=1}^{n} b c_{j}\left(\begin{array}{l}
\left|\left(\underline{\nu}_{D M\left(N^{*}\right)^{(l)}}\right)-\left(\underline{\nu}_{N^{*} I S^{(l)}}\right)\right|^{\lambda}+\left|\left(\underline{\eta}_{D M\left(N^{*}\right)^{(l)}}\right)-\left(\underline{\eta}_{N^{*} I S^{(l)}}\right)\right|^{\lambda} \\
\left|\left(\bar{\nu}_{D M\left(N^{*}\right)^{(l)}}\right)-\left(\bar{\nu}_{N^{*} I S^{(l)}}\right)\right|^{\lambda}+\left|\left(\bar{\eta}_{D M\left(N^{*}\right)^{(l)}}\right)-\left(\bar{\eta}_{\left.N^{*} I S^{(l)}\right)}\right)\right|^{\lambda}+
\end{array}\right)\right)^{\frac{1}{\lambda}}
\end{aligned}
$$

and for each $i=1,2, \ldots, m$.

Step-(4d): For each DM's the revised closeness indices denoted by $\left(R \dot{C}^{*} I_{i}\right)$ are calculated as follows:

$$
R \dot{C}^{*} I_{i}^{(l)}=\frac{D I S_{i}^{-(l)}}{D I S_{i}^{+(l)}+D I S_{i}^{-(l)}}
$$

Step-(5): Using the DMs weights, compute the final revised closeness indices $\left(F R \dot{C}^{*} I s\right)$ as follows:

$$
F R \dot{C}^{*} I_{i}=\sum_{l=1}^{e} b^{(l)} \cdot R \dot{C}^{*} I_{i}^{(l)}
$$

In ascending order, rank the measured $\left(F R \dot{C}^{*} I s\right)$ values; the alternative with the greatest value is our best choice.

\section{Numerical Application of the Proposed EXtended TOPSiS METHOD}

Now a days we are facing many problems like selection of a robot for a particular industrial application has always been a crucial issue due to the market's availability of various types of industrial robots with varying capacities, functionality, facilities, and specifications. For this we will present MCGDM a practical example to find the best optimal solution for selecting the different types of industrial robots.

Let us suppose there are four different types of industrial robots $\Upsilon=\left\{p_{1}^{*}, p_{2}^{*}, p_{3}^{*}, p_{3}^{*}\right\}$ with different features and there are three professional experts $\check{D}_{g}(g=1,2,3)$ having unknown weights vectors $b^{(l)}$. The experts assessed these four robots concerning the five criteria $C=\left\{c_{1}^{*}, c_{2}^{*}, c_{3}^{*}, c_{4}^{*}, c_{5}^{*}\right\}$ with unknown weight vector $b_{c_{j}}$ which are given as under

\section{1) Performance (Static and Dynamic)}

A robot's performance characteristics are divided into two categories: static and dynamic. The values given under steady state conditions are called static characteristics, while dynamic characteristics refer to the robot's time-dependent behavior.

\section{2) Robotic Architecture}


The geometry and movements needed to push around the robot's surroundings are important in the design and analysis of the robot.

\section{3) Operating Environment}

Robotic systems must operate in difficult and unpredictable settings, so the ability to communicate with and cope with the environment, whether on land, underwater, in the air, underground, or in space, is a vital skill.

\section{4) Instrumentation and Control Systems}

These are characteristics that are in charge of making important decisions based on input values from sensors and transducers, as well as monitoring and calculating the quantities of controllable parameter values.

\section{5) General and Physical}

These characteristics are a mix of cost-effective and desirable technological features relevant to a robot's efficiency and quality that aren't needed to complete any task but are extremely useful during installation and/or operation.

Now, in the form of IFRVs, the DM's assessed their assessment report for each $p_{i}^{*}$ in the form of IFRVs against the corresponding criteria are as follow in Table $1(\mathrm{a})(\mathrm{b})-3(\mathrm{a})(\mathrm{b})$.

Table-1(a): IFR evaluation information $\check{D}_{1}$

\begin{tabular}{llll}
\hline \hline & $c_{1}^{*}$ & $c_{2}^{*}$ & $c_{3}^{*}$ \\
\hline \hline$p_{1}^{*}$ & $((.5, .2),(.7, .1))$ & $((.7, .1),(.6, .3))$ & $((.6, .2),(.3, .2))$ \\
$p_{2}^{*}$ & $((.4, .1),(.7, .3))$ & $((.5, .2),(.3, .2))$ & $((.5, .2),(.8, .2))$ \\
$p_{3}^{*}$ & $((.5, .3),(.6, .3))$ & $((.5, .4),(.6, .3))$ & $((.7, .2),(.6, .2))$ \\
$p_{4}^{*}$ & $((.8, .1),(.5, .4))$ & $((.6, .4),(.4, .3))$ & $((.4, .3),(.6, .4))$ \\
\hline
\end{tabular}

Table-1(b): IFR evaluation information $\check{D}_{1}$

\begin{tabular}{lll}
\hline \hline & $c_{4}^{*}$ & $c_{5}^{*}$ \\
\hline \hline$p_{1}^{*}$ & $((.7, .3),(.6, .3))$ & $((.5, .2),(.3, .1))$ \\
$p_{2}^{*}$ & $((.6, .3),(.6, .2))$ & $((.3, .2),(.5, .3))$ \\
$p_{3}^{*}$ & $((.6, .4),(.2, .3))$ & $((.5, .3),(.3, .2))$ \\
$p_{4}^{*}$ & $((.5, .1),(.4, .5))$ & $((.8, .3),(.5, .2))$ \\
\hline
\end{tabular}

Table-2(a): IFR evaluation information $\check{D}_{2}$

\begin{tabular}{llll}
\hline \hline & $c_{1}^{*}$ & $c_{2}^{*}$ & $c_{3}^{*}$ \\
\hline \hline$p_{1}^{*}$ & $((.7, .1),(.3, .1))$ & $((.8, .1),(.5, .2))$ & $((.7, .2),(.8, .1))$ \\
$p_{2}^{*}$ & $((.5, .1),(.5, .4))$ & $((.7, .2),(.6, .3))$ & $((.5, .1),(.4, .5))$ \\
$p_{3}^{*}$ & $((.6, .2),(.2, .6))$ & $((.4, .3),(.3, .6))$ & $((.3, .4),(.7, .2))$ \\
$p_{4}^{*}$ & $((.4, .5),(.6, .3))$ & $((.6, .4),(.5, .3))$ & $((.2, .3),(.9, .1))$ \\
\hline
\end{tabular}

Table-2(b): IFR evaluation information $\check{D}_{2}$

\begin{tabular}{lll}
\hline \hline & $c_{4}^{*}$ & $c_{5}^{*}$ \\
\hline \hline$p_{1}^{*}$ & $((.3, .5),(.6, .2))$ & $((.8, .2),(.6, .2))$ \\
$p_{2}^{*}$ & $((.4, .5),(.3, .5))$ & $((.7, .1),(.5,0.3))$ \\
$p_{3}^{*}$ & $((.2, .6),(.4, .2))$ & $((.9, .1),(.2, .3))$ \\
$p_{4}^{*}$ & $((.6, .3),(.5, .1))$ & $((.5, .4),(.6, .3))$ \\
\hline
\end{tabular}


Table-3(a): IFR evaluation information $\check{D}_{3}$

\begin{tabular}{llll}
\hline \hline & $c_{1}^{*}$ & $c_{2}^{*}$ & $c_{3}^{*}$ \\
\hline \hline$p_{1}^{*}$ & $((.8, .2),(.3, .2))$ & $((.7, .3),(.5, .4))$ & $((.8, .2),(.5, .4))$ \\
$p_{2}^{*}$ & $((.6, .3),(.4, .5))$ & $((.6, .4),(.6, .3))$ & $((.4, .3),(.3, .5))$ \\
$p_{3}^{*}$ & $((.3, .4),(.6, .2))$ & $((.8, .2),(.5, .5))$ & $((.5, .4),(.2, .5))$ \\
$p_{4}^{*}$ & $((.4, .2),(.6, .4))$ & $((.4, .3),(.3, .7))$ & $((.3, .4),(.4, .3))$ \\
\hline
\end{tabular}

Table-3(b): IFR evaluation information $\check{D}_{3}$

\begin{tabular}{lll}
\hline \hline & $c_{4}^{*}$ & $c_{5}^{*}$ \\
\hline \hline$p_{1}^{*}$ & $((.6, .3),(.5, .3))$ & $((.9, .1),(.6, .2))$ \\
$p_{2}^{*}$ & $((.4, .6),(.6, .2))$ & $((.7, .3),(.5, .3))$ \\
$p_{3}^{*}$ & $((.7, .3),(.4, .5))$ & $((.5, .4),(.5, .2))$ \\
$p_{4}^{*}$ & $((.4, .1),(.6, .3))$ & $((.6, .2),(.6, .3))$ \\
\hline
\end{tabular}

Now we use a new formed approach of IFERWA operator to achieve the best possible solution for selecting different types of robots based on the extended TOPSIS method. The step-wise decision algorithm are as under.

Step-(1): Since all of the criteria are of the same kind, so there is no need to normalize them. Then the above three matrices are consider to be normalized matrices.

Step-(2a): $G^{*} D \tilde{I} S$ matrix is computed as follows:

Table-4(a): $G^{*} D \tilde{I} S^{\prime}$

\begin{tabular}{|c|c|c|c|}
\hline$c_{1}^{*}$ & & $c_{2}^{*}$ & $c_{3}^{*}$ \\
\hline & $(.6845, .1598)$ & $((.7368, .1464)$, & $\int((.7091, .2004)$, \\
\hline$p_{1}$ & $(.4581, .1268)$ & $(.5347, .2909)$ & $(.5729, .2043)$ \\
\hline & $(.5041, .1464)$ & $(.6060, .2546)$ & $(.4675, .1839)$ \\
\hline$p_{2}$ & $(.5461, .3940)$ & $(.5115, .2632)$ & $(.5441, .3759)$ \\
\hline & $(.4752, .2909)$ & $(.5979, .2909)$ & \}$(.5189, .3209)$ \\
\hline$p_{3}$ & $(.4847, .3384)$ & $(.4752, .4539)$ & $(.5278, .2767)$ \\
\hline & $(.5703, .2225)$ & $(.5390, .3646)$, & $(.3019, .3313)$ \\
\hline$p_{4}$ & $(.5680, .3646)$ & $(.4029, .4080)$ & $(.6974, .2343)$ \\
\hline
\end{tabular}

Table-4(b): $G^{*} D \tilde{I} \dot{S}$

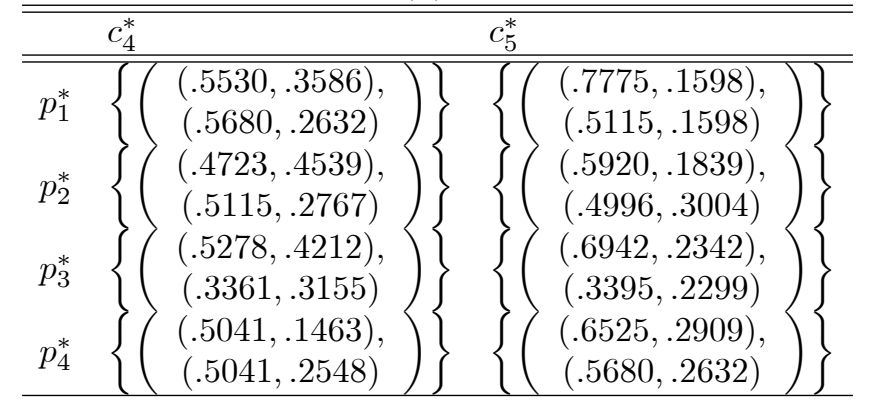


Step-(2b): Here we find the $G^{*} \tilde{R} \tilde{I} D, G^{*} \tilde{L} \tilde{I} D$ which are given below:

Table-5(a): $G^{*} R \tilde{I} D$

\begin{tabular}{llll}
\hline \hline & $c_{1}^{*}$ & $c_{2}^{*}$ & $c_{3}^{*}$ \\
\hline \hline$p_{1}^{*}$ & $((.5,0.2),(.7, .1))$ & $((.8,0.1),(.5, .2))$ & $((.7, .2),(.8, .1))$ \\
$p_{2}^{*}$ & $((.4,0.1),(.7, .3))$ & $((.7,0.2),(.6, .3))$ & $((.5, .2),(.8, .2))$ \\
$p_{3}^{*}$ & $((.5,0.3),(.6, .3))$ & $((.8,0.2),(.5, .5))$ & $((.7, .2),(.6, .2))$ \\
$p_{4}^{*}$ & $((.8,0.1),(.5, .4))$ & $((.6,0.4),(.5, .3))$ & $((.2, .3),(.9, .1))$ \\
\hline
\end{tabular}

Table-5(b): $G^{*} R \tilde{I} D$

\begin{tabular}{lll}
\hline \hline & $c_{4}^{*}$ & $c_{5}^{*}$ \\
\hline \hline$p_{1}^{*}$ & $((.7, .3),(.6, .3))$ & $((.9, .1),(.6, .2))$ \\
$p_{2}^{*}$ & $((.6, .3),(.6, .2))$ & $((.7, .1),(.5, .3))$ \\
$p_{3}^{*}$ & $((.7, .3),(.4, .5))$ & $((.9, .1),(.2, .3))$ \\
$p_{4}^{*}$ & $((.6, .3),(.5, .1))$ & $((.8, .3),(.5, .2))$ \\
\hline
\end{tabular}

Table-6(a): $G^{*} L \tilde{I} D$

\begin{tabular}{llll}
\hline \hline & $c_{1}^{*}$ & $c_{2}^{*}$ & $c_{3}^{*}$ \\
\hline \hline$p_{1}^{*}$ & $((.8, .2),(.3, .2))$ & $((.7, .3),(.5, .4))$ & $((.6, .2),(.3, .2))$ \\
$p_{2}^{*}$ & $((.6, .3),(.4, .5))$ & $((.5, .2),(.3, .2))$ & $((.4, .3),(.3, .5))$ \\
$p_{3}^{*}$ & $((.6, .2),(.2, .6))$ & $((.5, .4),(.6, .3))$ & $((.5, .4),(.2, .5))$ \\
$p_{4}^{*}$ & $((.4, .5),(.6, .3))$ & $((.4, .3),(.3, .7))$ & $((.3, .4),(.4, .3))$ \\
\hline
\end{tabular}

Table-6(b): $G^{*} \dot{L} \tilde{I} D$

\begin{tabular}{lll}
\hline \hline & $c_{4}^{*}$ & $c_{5}^{*}$ \\
\hline \hline$p_{1}^{*}$ & $((.3, .5),(.6, .2))$ & $((.5, .2),(.3, .1))$ \\
$p_{2}^{*}$ & $((.4, .5),(.3, .5))$ & $((.3, .2),(.5, .3))$ \\
$p_{3}^{*}$ & $((.2, .6),(.4, .2))$ & $((.5, .3),(.3, .2))$ \\
$p_{4}^{*}$ & $((.5, .1),(.4, .5))$ & $((.5, .4),(.6, .3))$ \\
\hline
\end{tabular}

(1) Step-(2c): Using equation 4.1, for $\lambda=2, D G^{*} D \tilde{I} \dot{S}, D G^{*} \hat{R} \tilde{I} D$ and $D G^{*} L \tilde{I} D$ are computed as follows:

Table-7 $D G^{*} D \tilde{I} S$ Matrix

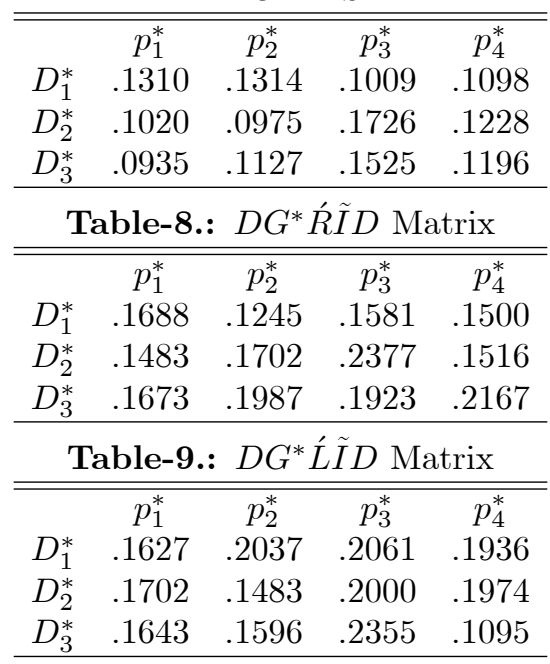


Step-(2d): The following are the closeness indices (CIs):

\begin{tabular}{ccc}
\hline \hline$\dot{C}^{*} I^{(1)}$ & $\dot{C}^{*} I^{(2)}$ & $\dot{C}^{*} I^{(3)}$ \\
.7429 & .7420 & .7512 \\
\hline
\end{tabular}

Step-(2e): In this step, the following equation is used to determine the weights of DMs:

\begin{tabular}{ccc}
\hline \hline$b^{(1)}$ & $b^{(2)}$ & $b^{(3)}$ \\
.33224 & .33182 & .33594 \\
\hline
\end{tabular}

Step-(3a): Group revised decision $R G^{*} D \tilde{I} S$ are computed as follows:

Table-10(a): $R G^{*} D \tilde{I} S$

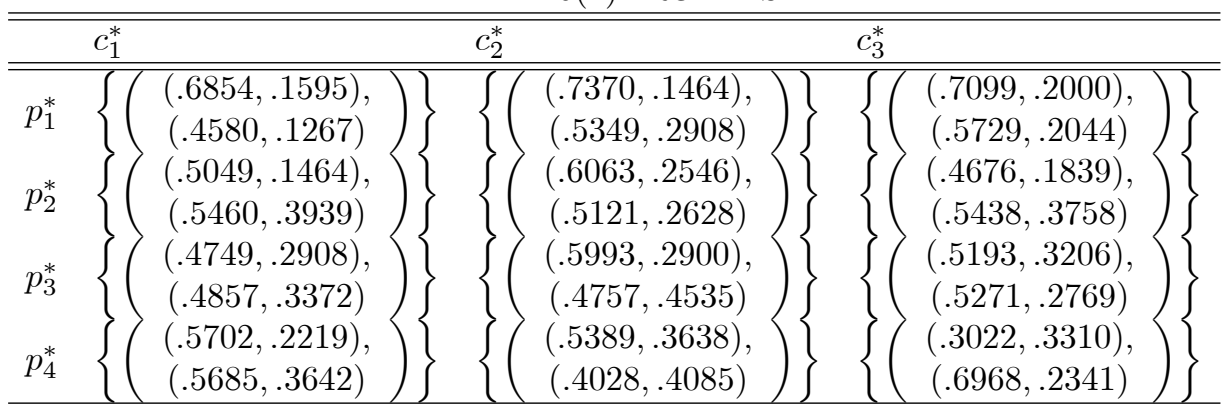

Table-10(b): $R G^{*} D \tilde{I} S$

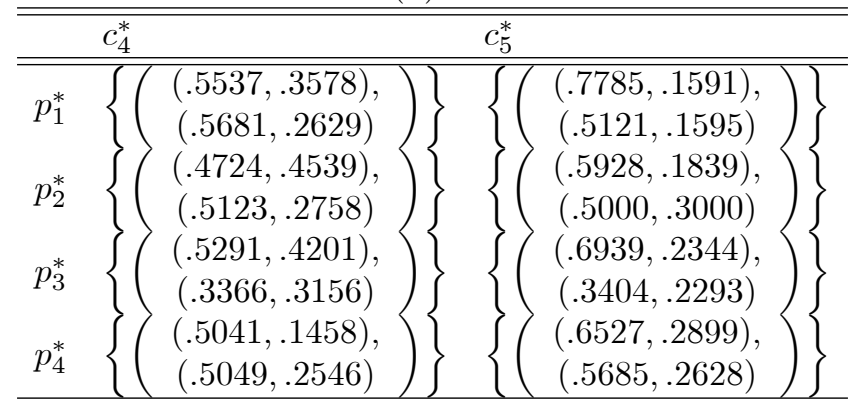

Step-(3b): The Intuitionistic fuzzy rough entropy measure for each attribute is calculated as follows:

\begin{tabular}{ccccc}
\multicolumn{5}{c}{ Table-11 } \\
\hline \hline$E C_{1}$ & $E C_{2}$ & $E C_{3}$ & $E C_{3}$ & $E C_{4}$ \\
\hline \hline .3325 & .3087 & .3199 & .3660 & .2891 \\
\hline
\end{tabular}

Step-3(c): Criteria weight are calculated as follows:

Table-12

\begin{tabular}{lllll}
\hline \hline$b c_{1}$ & $b c_{2}$ & $b c_{3}$ & $b c_{4}$ & $b c_{5}$ \\
\hline \hline .197 & .204 & .201 & .188 & 0.210 \\
\hline
\end{tabular}


Step-(4a): Here, we determined the weighted normalized decision matrices given in following Tables 13(a), 14(a), 15(a):

Table-13(a): Weighted Normalized $\check{D}_{1}$ matrix $\left(D M\left(N^{*}\right)_{i j}^{(1)}\right)$

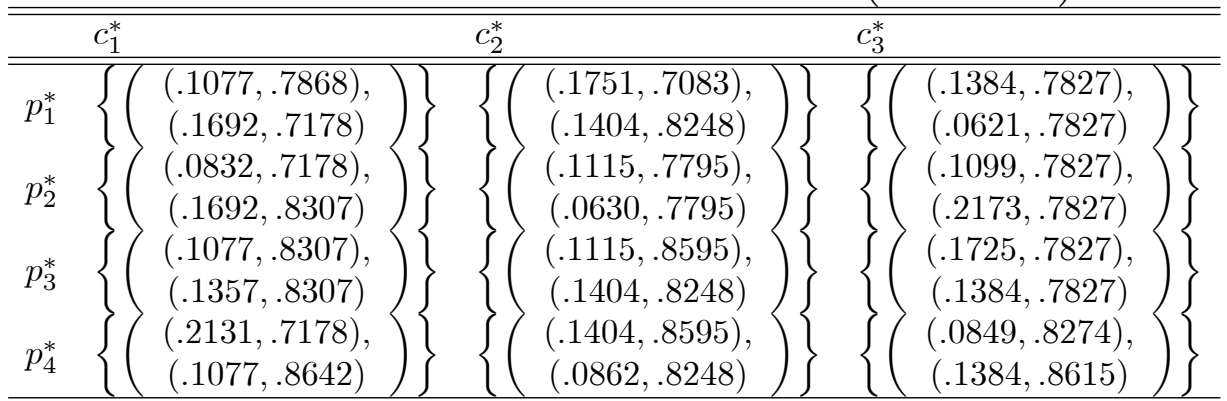

Table-13(b): Weighted Normalized $\check{D}_{1}$ matrix $\left(D M\left(N^{*}\right)_{i j}^{(1)}\right)$

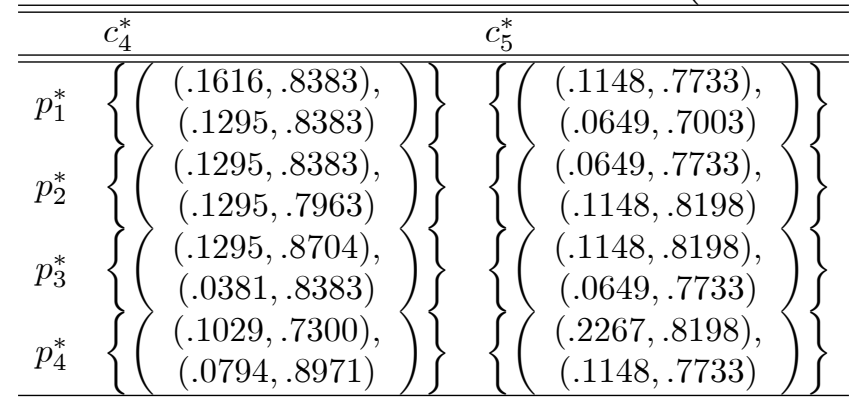

Table-14(a): Weighted Normalized $\check{D}_{2}$ matrix $\left(D M\left(N^{*}\right)_{i j}^{(2)}\right)$

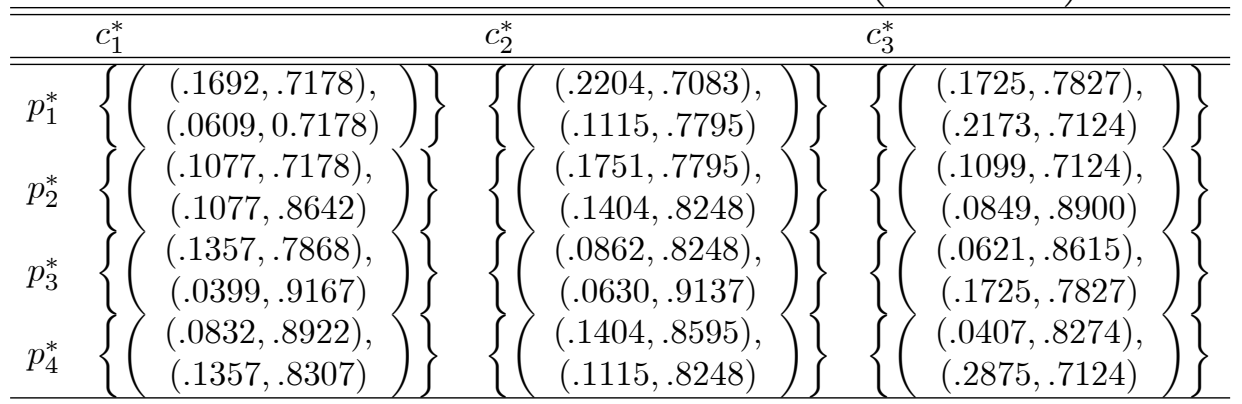

Table-14(b): Weighted Normalized $\check{D}_{2}$ matrix $\left(D M\left(N^{*}\right)_{i j}^{(2)}\right)$

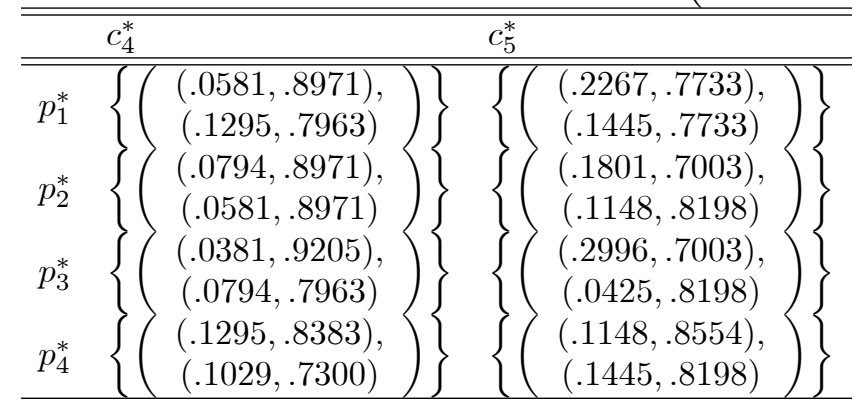


Table-15(a): Weighted Normalized $\check{D}_{3}$ matrix $\left(D M\left(N^{*}\right)_{i j}^{(3)}\right)$

\begin{tabular}{|c|c|c|c|}
\hline & & $c_{2}^{*}$ & $c_{3}^{*}$ \\
\hline & $(.2131, .7868)$ & $\int((.1751, .8248)$, & $(.2173,0.7827)$, \\
\hline$p_{1}^{1}$ & $(.0609, .7868)$ & $(.1115, .8595)$ & $(.1099, .8615)$ \\
\hline & $(.1357, .8307)$ & $(.1404, .8595)$ & $(.0849, .8274)$ \\
\hline$p_{2}$ & $(.0832, .8922)$ & $(.1404, .8248)$ & $(.0621, .8900)$ \\
\hline & $(.0609, .8642)$ & $(.2204, .7795)$ & $(.1099, .8615)$ \\
\hline$p_{3}$ & $(.1357, .7868)$ & $(.1115, .8884)$ & $(.0407, .8900)$ \\
\hline & $(.0832, .7868)$ & $(.0862, .8248)$, & $(.0621, .8615)$ \\
\hline$p_{4}$ & $(.1357, .8642)$ & $(.0630, .9369)$ & $(.0849, .8274)$ \\
\hline
\end{tabular}

Table-15(b): Weighted Normalized $\check{D}_{3}$ matrix $\left(D M\left(N^{*}\right)_{i j}^{(3)}\right)$

\begin{tabular}{|c|c|c|}
\hline & & \\
\hline & $(.1295, .8383)$ & $(.2996, .7003)$, \\
\hline$p_{1}$ & $(.1029, .8383)$ & $(.1445, .7733)$ \\
\hline & $(.0794, .9205)$ & $(.1801, .8198)$, \\
\hline$p_{2}$ & $(.1295, .7963)$ & $(.1148, .8198)$ \\
\hline & $(.1616, .8383)$, & $(.1148, .8554)$, \\
\hline$p_{3}^{*}$ & $(.0794, .8971)$ & $(.1148, .7733)$ \\
\hline & $(.0794, .7300)$, & $(.1445, .7733)$, \\
\hline$p_{4}$ & $(.1295, .8383)$ & $(.1445, .8198)$ \\
\hline
\end{tabular}

Step-(4b): $P^{*} I S^{(l)}$ and $N^{*} I S^{(l)}$ for each DM's of weighted normalized matrix are determined as:

Table-16(a): $P^{*} I S^{(l)}$ for each DM's

\begin{tabular}{llll}
\hline \hline & $c_{1}^{*}$ & $c_{2}^{*}$ & $c_{3}^{*}$ \\
\hline \hline$P^{*} I S^{(1)}$ & $\left\{\left(\begin{array}{c}(.1077, .7868), \\
(.1692, .7178) \\
(.1692, .7178), \\
P^{*} I S^{(2)}\end{array}\right)\right\}$ & $\left\{\left(\begin{array}{c}(.1751, .7083), \\
(.1404, .8248)\end{array}\right)\right\}$ & $\left.\left\{\begin{array}{l}(.1099, .7827), \\
(.2173, .7827) \\
(.2204, .7083), \\
(.1115, .7795)\end{array}\right)\right\}$ \\
$P^{*} I S^{(3)}$ & $\left\{\left(\begin{array}{l}.1725, .7827), \\
(.2131,0.7868), \\
(.0609, .7868)\end{array}\right)\right\}$ & $\left.\left\{\begin{array}{l}(.2173, .7124) \\
(.2204, .7795), \\
(.1115, .8884)\end{array}\right)\right\}$ & $\left.\left\{\begin{array}{l}(.2173, .7827), \\
(.1099, .8615)\end{array}\right)\right\}$ \\
\hline
\end{tabular}

Table-16(b): $P^{*} I S^{(l)}$ for each DM's

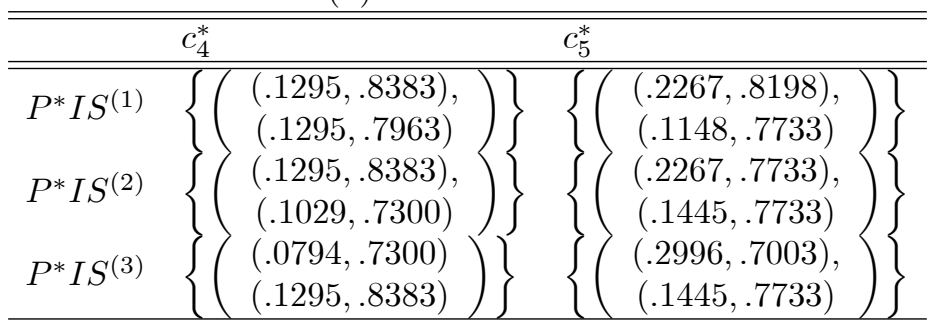


Table-17(a): $N^{*} I S^{(l)}$ for each DM's

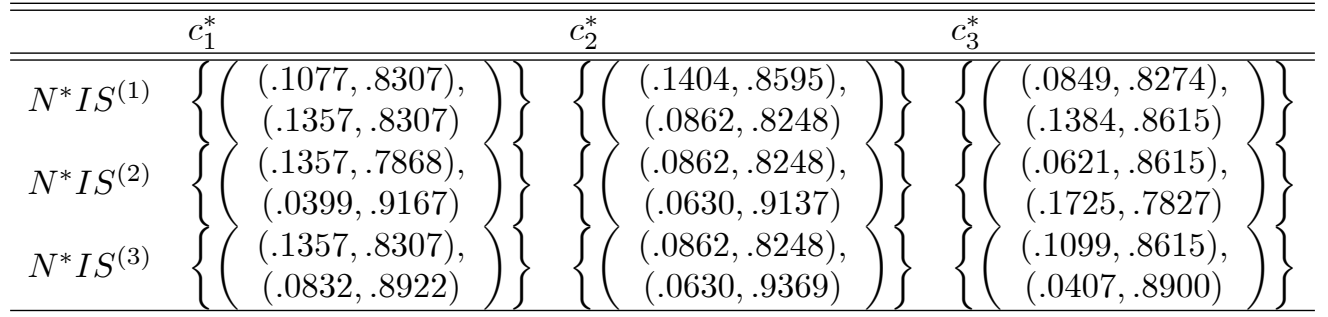

Table-17(b): $N^{*} I S^{(l)}$ for each DM's

\begin{tabular}{|c|c|c|}
\hline & $c_{4}^{*}$ & \\
\hline$N^{*} I S^{(1)}$ & $\left\{\left(\begin{array}{c}(.1295, .8704), \\
(.0381, .8383)\end{array}\right.\right.$ & $\begin{array}{c}(.0649, .7733) \\
(.1148, .8198)\end{array}$ \\
\hline$N^{*} I S^{(2)}$ & $\left\{\begin{array}{c}(.0794, .8971), \\
(.0581, .8971)\end{array}\right.$ & $\begin{array}{l}(.1148, .8554) \\
(.1445, .8198)\end{array}$ \\
\hline$N^{*} I S^{(3)}$ & $\left\{\left(\begin{array}{c}(.0794, .9205), \\
(.1295, .7963)\end{array}\right)\right.$ & $\begin{array}{c}(.1148, .8554) \\
(.1148, .7733)\end{array}$ \\
\hline
\end{tabular}

Step-(4c): The attributes (criteria) weights have been calculated. The weighted distances of weighted $D M\left(N^{*}\right)_{i j}^{(l)}$ from $P^{*} I S^{(l)}$ and $N^{*} I S^{(l)}$ are denoted by $D I S_{i}^{+(l)}$ and $D I S_{i}^{-(l)}$, respectively, which are calculated as follows:

Table-18

\begin{tabular}{lllll}
\hline \hline & $p_{1}^{*}$ & $p_{2}^{*}$ & $p_{3}^{*}$ & $p_{4}^{*}$ \\
\hline \hline$D I S_{i}^{+(1)}$ & .0179 & .0203 & .0258 & .0362 \\
$D I S_{i}^{+(2)}$ & .0113 & .0507 & .0408 & .0340 \\
$D I S_{i}^{+(3)}$ & .0138 & .0334 & .0309 & .0310 \\
\hline
\end{tabular}

Table-19

\begin{tabular}{lllll}
\hline \hline & $p_{1}^{*}$ & $p_{2}^{*}$ & $p_{3}^{*}$ & $p_{4}^{*}$ \\
\hline \hline$D I S_{i}^{-(1)}$ & .0313 & .0273 & .0215 & .0235 \\
$D I S_{i}^{-(2)}$ & .0402 & .0364 & .0160 & .0343 \\
$D I S_{i}^{-(3)}$ & .0291 & .0164 & .0264 & .0263 \\
\hline
\end{tabular}

Step-(4d): For each DM's, revised closeness indices $\left(R \dot{C}^{*} I s\right)$ are calculated as follows: 
Table-18

\begin{tabular}{lllll}
\hline \hline & $p_{1}^{*}$ & $p_{2}^{*}$ & $p_{3}^{*}$ & $p_{4}^{*}$ \\
\hline \hline$R \dot{C}^{*} I_{i}^{(1)}$ & .6365 & .5733 & .4549 & .3935 \\
$R \dot{C}^{*} I_{i}^{(2)}$ & .7804 & .4179 & .2815 & .5022 \\
$R \dot{C}^{*} I_{i}^{(3)}$ & .6785 & .3294 & .4613 & .4596 \\
\hline
\end{tabular}

Step-(5): Using the DM's weights, the final revised closeness indices $\left(F R \dot{C}^{*} I_{i}\right)$ are calculated as follows:

Table-19:

\begin{tabular}{ccccc}
\hline \hline Alternatives & $P_{1}^{*}$ & $P_{2}^{*}$ & $P_{3}^{*}$ & $P_{4}^{*}$ \\
\hline \hline$F R \dot{C}^{*} I_{i}$ & .6984 & .4400 & .3995 & .4517 \\
\hline
\end{tabular}

As a result, based on the attributes, $P_{1}^{*}$ is our best choice.

\section{Comparative Study}

In this part, we will discuss the advantages of the our new proposed method Extended TOPSIS which are demonstrated by comparing its characteristics with different MCGDM methods. The PIS and NIS are used in the Extended TOPSIS system. The best alternative is PIS because of its superior value and NIS because of its inferior value. A comparison of our investigated IFR TOPSIS method with some existing methods in background has been made to prove the supremacy of our investigative IFR TOPSIS method (see [12, 34, 57, 58]) which are discussed in the following subsection.

7.1. Comparison with IFWA. In this subsection we can compare our new proposed work with the existing methods. As in existing methods Xu [12] take the values in the form IFSs, and defined the averaging aggregation operators such as IFWA and IFOWA, to aggregate the information with IFRVs, when applied to decision making based on intuitionistic fuzzy knowledge, this makes the decision results more precise and realistic. But here in our paper we take the data in the form of IFRSs and used basic concept of Einstein T-norm and T-conorm to introduced a new aggregation operators like IFREWA, IFREOWA and IFREHWA to aggregate the MCGDM issues. Further more we have used these aggregation operators for solving the MCGDM in form of IFRSs environment with all unknown information about the weights of criteria and DMs. From Table-20 it is clear that the existing aggregation operators are incapable to solve the formed illustrated example with IFRVs. So by comparing this proposed work with the existing work we can see that our proposed work is more accurate and more applicable than existing methods.

Table-20:

\begin{tabular}{l||cccc||c}
\hline \hline \multicolumn{1}{c||}{ Approaches } & \multicolumn{4}{c||}{ Score values } & Ranking \\
\hline IFWA [12] & $\times$ & $\times$ & $\times$ & $\times$ & $\times$ \\
\hline IFREWA (Extended TOPSIS) & .6984 & .4400 & .3995 & .4517 & $p_{1}^{*}>p_{4}^{*}>p_{2}^{*}>p_{3}^{*}$ \\
\hline
\end{tabular}


7.2. Comparison with IFDWA. In this subsection we also compare our proposed work with the another existing methods. As in existing methods M. R. Seikh et al. [57] give the idea of Dombi T-norm and T-conorm to aggregate intuitionistic fuzzy knowledge. Also discussed there basic operational laws in the form of IFNs environment. On the basis of these operational laws, in existing method the aggregation operators were defined like IFDWA, IFDOWA, and IFDHA operators for aggregating the MCGDM problems under an intuitionistic fuzzy environment. But here in our paper we take the data in the form of IFRSs and used basic concept of Einstein T-norm and T-conorm we introduced a new aggregation operators like IFREWA, IFREOWA and IFREHWA to aggregate the MCGDM issues. Moreover we have used these aggregation operators for solving the MCGDM in form of IFRSs environment with all unknown information about the weights of criteria and DMs. It is clear from Table 21 that the current aggregation operators are incapable to solve the formed illustrated example with IFVs. So by comparing this proposed work with the existing work we can see that our new work is more accurate and more applicable than existing methods.

Table-21:

\begin{tabular}{l||cccc||c}
\hline \hline \multicolumn{1}{c||}{ Approaches } & \multicolumn{4}{c||}{ Score values } & Ranking \\
\hline \hline IFDWA [57] & $\times$ & $\times$ & $\times$ & $\times$ & $\times$ \\
\hline IFREWA (Extended TOPSIS) & .6984 & .4400 & .3995 & .4517 & $p_{1}^{*}>p_{4}^{*}>p_{2}^{*}>p_{3}^{*}$ \\
\hline
\end{tabular}

7.3. Comparison with IF-EDAS Method. We can compare our new proposed work with the existing methods in this subsection. Ghorabaee et al. [58] proposed an advance method of Evaluation depend on Distance from Average solution EDAS by taking the data in the form of IFS for multi-criteria inventory classification and also examined its step-wise algorithms. But here in this paper we can take the data in the form of IFRSs and used the idea of Einstein T-norm and T-conorm. Further we can also extend and proposed the new aggregation operators like IFREWA, IFREOWA and IFREHWA, to aggregate the MCGDM issues. Finally we have used these developed operators on new Extended TOPSIS method with all unknown information about weights of DMs and criteria for finding best options. It is clear that IF-EDAS method, are clearly incapable to solve the established illustrated example in the form of IFR information, as shown in Table-22. We can see that current methods lack IF knowledge, and this approach is incapable of solving and ranking the established example. As a result, our newly established system is more capable and reliable than current approaches which are shown as follows:

Table-22

\begin{tabular}{l||cccc||c}
\hline \hline \multicolumn{1}{c||}{ Approaches } & \multicolumn{4}{c||}{ Score values } & Ranking \\
\hline \hline IF-EDAS [58] & $\times$ & $\times$ & $\times$ & $\times$ & $\times$ \\
\hline IFREWA (Extended TOPSIS) & .6984 & .4400 & .3995 & .4517 & $p_{1}^{*}>p_{4}^{*}>p_{2}^{*}>p_{3}^{*}$ \\
\hline
\end{tabular}

7.4. Comparison with IFR-EDAS Method. We can compare our new proposed work with the existing methods in this subsection. In this existing methods Mehmood et [34] take the idea of IFRSs and defined its basic aggregation operators like IFRWA, IFROWA and IFRHWA also defined a new score and accuracy function. And applied these operators on new proposed method IFR-(EDAS) in 
form of IFRSs with all known informations about the criteria weights and DMs. The step-wise details were given in literature. But here in our paper we take the same data and used basic concept of Einstein t-norm and t-conorm to introduced a new aggregation operators like IFREWA, IFREOWA and IFREHWA to aggregate the MCGDM issues. Further more we used these aggregation operators on new proposed IFR-Extended TOPSIS method in form of IFRSs environments with unknown weights of criteria and DMs. From obtaining outcome shown in Table-23, $p_{1}^{*}$ is the best alternative, that's the same as given in [34].

Table-23

\begin{tabular}{l||llll||c}
\hline \hline \multicolumn{1}{c||}{ Approaches } & \multicolumn{4}{c||}{ Score values } & Ranking \\
\hline \hline IFRSs-EDAS [34] & 0.66 & 0.52 & 0.37 & 0.34 & $p_{1}^{*}>p_{2}^{*}>p_{3}^{*}>p_{4}^{*}$ \\
\hline IFREWA (Extended TOPSIS) & .6984 & .4400 & .3995 & .4517 & $p_{1}^{*}>p_{4}^{*}>p_{2}^{*}>p_{3}^{*}$ \\
\hline
\end{tabular}

Overall Ranking Table-24

\begin{tabular}{l||l|l|l|l||c}
\hline \multicolumn{1}{c||}{ Approaches } & \multicolumn{4}{c||}{ Score values } & Ranking \\
\cline { 2 - 7 } IFWA [12] & $\times$ & $\times$ & $\times$ & $\times$ & $\times$ \\
\hline IFDWA [57] & $\times$ & $\times$ & $\times$ & $\times$ & $\times$ \\
\hline IF-EDAS [58] & $\times$ & $\times$ & $\times$ & $\times$ & $\times$ \\
\hline IFRSs-EDAS [34] & 0.66 & 0.52 & 0.37 & 0.34 & $p_{1}^{*}>p_{2}^{*}>p_{3}^{*}>p_{4}^{*}$ \\
\hline IFREWA (Extended TOPSIS) & .6984 & .4400 & .3995 & .4517 & $p_{1}^{*}>p_{4}^{*}>p_{2}^{*}>p_{3}^{*}$ \\
\hline
\end{tabular}

7.5. Results and Discussion. As a results given in Tables-24, we conclude that current approache like IF-EDAS method [58], and several aggregation operators $[12,57]$ are incapable to solve the established illustrated example in the form of IFR setting except the IFR-EDAS. In comparison with IFR-EDAS given in Table-24, the information is provided by the DM's in the form of IFRSs we applied Extended TOPSIS approach with all unknown details about the weights of criteria and DMs. As a result, $p_{1}^{*}$ is the best option, that is the same as mentioned in [34]. We can see most of the current methods lack rough details, and these are unable to solve or rank the established example.

Hence, the presented method is more reliable, advisable, efficient, and generalized for solving MCGDM problems with totally unknown DM's and criteria knowledge.

\section{Conclusion}

The MCGDM has a massive potential and discipline process for improving and evaluating various conflicting criteria in all aspects of DM in order to obtain more appropriate and realistic DM outcome. In DM issues, the effective learning regarding a particular fact is often unknown, making the decision-making task more complicated and dynamic. RSs and intuitionistic fuzzy sets are general mathematical method that can easily handle ambiguous and imprecise knowledge. When there are several conflict criteria in MCGDM problems, the TOPSIS approach plays an important part in the success. In this article we established a new Extended TOPSIS approach in the form of IFR information which is based on Einstein operators, with all unknown information about weights of criteria and DM's. For this we give the idea IFR-Einstein aggregation operators like IFREWA, IFREOWA and IFREHA. To create the IFR entropy weight framework for calculating the criteria weights with in IFR data, a new GDM-based IFR entropy measure is presented. In last steps, aggregation is conducted by using determined DMs weights to achieve 
the final ranking, to prevent the loss of knowledge base in this method. Finally, examples are given to show the technique's potential application and advantage. Furthermore, the established approach can be expanded for future research by incorporating other existing fuzzy sets and applying them to various MCGDM problems involving unknown DM and criteria weights.

\section{REFERENCES}

[1] Garg, H., 2016. A new generalized Pythagorean fuzzy information aggregation using Einstein operations and its application to decision making. International Journal of Intelligent Systems, 31(9), pp.886-920.

[2] Ashraf, S., Mahmood, T., Abdullah, S. and Khan, Q., 2019. Different approaches to multicriteria group decision making problems for picture fuzzy environment. Bulletin of the Brazilian Mathematical Society, New Series, 50(2), pp.373-397.

[3] Rahman, K. and Abdullah, S., Some new generalized interval-valued Pythagorean fuzzy aggregation operators using Einstein t-norm and t-conorm. Journal of Intelligent \& Fuzzy Systems, (Preprint), pp.1-22.

[4] Ashraf, S., Abdullah, S. and Smarandache, F., 2019. Logarithmic Hybrid Aggregation Operators Based on Single Valued Neutrosophic Sets and Their Applications in Decision Support Systems. Symmetry, 11(3), p.364.

[5] Khan, M.J., Kumam, P., Ashraf, S. and Kumam, W., 2019. Generalized Picture Fuzzy Soft Sets and Their Application in Decision Support Systems. Symmetry, 11(3), p.415.

[6] Ashraf, S., Abdullah, S., Mahmood, T. and Aslam, M., 2019. Cleaner Production Evaluation in Gold Mines Using Novel Distance Measure Method with Cubic Picture Fuzzy Numbers. International Journal of Fuzzy Systems, pp.1-14

[7] L. A. Zadeh, "Fuzzy sets," Inf Control., Vol. 8, no. 3, pp. 338-353, 1965.

[8] Xu, Z., Chen, J. and Wu, J., 2008. Clustering algorithm for intuitionistic fuzzy sets. Information Sciences, 178(19), pp.3775-3790.

[9] Hong, D.H. and Choi, C.H., 2000. Multicriteria fuzzy decision-making problems based on vague set theory. Fuzzy sets and systems, 114(1), pp.103-113.

[10] De, S.K., Biswas, R. and Roy, A.R., 2001. An application of intuitionistic fuzzy sets in medical diagnosis. Fuzzy sets and Systems, 117(2), pp.209-213.

[11] K. T. Atanassov, "Intuitionistic fuzzy sets," Fuzzy Sets Syst., vol. 20, no, 1, pp. 87-96, 1986.

[12] Xu, Z., 2007. Intuitionistic fuzzy aggregation operators. IEEE Transactions on fuzzy systems, 15(6), pp.1179-1187.

[13] Xu, Z. and Yager, R.R., 2006. Some geometric aggregation operators based on intuitionistic fuzzy sets. International journal of general systems, 35(4), pp.417-433.

[14] Ali, M.I., Feng, F., Mahmood, T., Mahmood, I. and Faizan, H., 2019. A graphical method for ranking Atanassov's intuitionistic fuzzy values using the uncertainty index and entropy. International Journal of Intelligent Systems, 34(10), pp.2692-2712.

[15] He, Y., Chen, H., He, Z. and Zhou, L., 2015. Multi-attribute decision making based on neutral averaging operators for intuitionistic fuzzy information. Applied Soft Computing, 27, pp.64-76.

[16] He, Y., Chen, H., Zhou, L., Liu, J. and Tao, Z., 2014. Intuitionistic fuzzy geometric interaction averaging operators and their application to multi-criteria decision making. Information Sciences, 259, pp.142-159.

[17] Wan, S.P., Xu, G.L. and Dong, J.Y., 2020. An Atanassov intuitionistic fuzzy programming method for group decision making with interval-valued Atanassov intuitionistic fuzzy preference relations. Applied Soft Computing, 95, p.106556.

[18] Wan, S. and Dong, J., 2021. A novel extension of best-worst method with intuitionistic fuzzy reference comparisons. IEEE Transactions on Fuzzy Systems.

[19] Zhao, H., Xu, Z., Ni, M. and Liu, S., 2010. Generalized aggregation operators for intuitionistic fuzzy sets. International journal of intelligent systems, 25(1), pp.1-30.

[20] Wang, W. and Liu, X., 2011. Intuitionistic fuzzy geometric aggregation operators based on Einstein operations. International Journal of Intelligent Systems, 26(11), pp.1049-1075. 
[21] Wang, J.Q. and Zhang, H.Y., 2012. Multicriteria decision-making approach based on Atanassov's intuitionistic fuzzy sets with incomplete certain information on weights. IEEE Transactions on Fuzzy Systems, 21(3), pp.510-515.

[22] Pawlak, Z., 1982. Rough sets. International journal of computer \& information sciences, 11(5), pp.341-356.

[23] Dubois, D. and Prade, H., 1990. Rough fuzzy sets and fuzzy rough sets. International Journal of General System, 17(2-3), pp.191-209.

[24] Cornelis, C., De Cock, M. and Kerre, E.E., 2003. Intuitionistic fuzzy rough sets: at the crossroads of imperfect knowledge. Expert systems, 20(5), pp.260-270.

[25] Zhou, L. and Wu, W.Z., 2008. On generalized intuitionistic fuzzy rough approximation operators. Information Sciences, 178(11), pp.2448-2465.

[26] Zhou, L. and Wu, W.Z., 2011. Characterization of rough set approximations in Atanassov intuitionistic fuzzy set theory. Computers \& Mathematics with Applications, 62(1), pp.282296.

[27] Bustince, H. and Burillo, P., 1996. Structures on intuitionistic fuzzy relations. Fuzzy sets and systems, 78(3), pp.293-303.

[28] Zhang, X., Zhou, B. and Li, P., 2012. A general frame for intuitionistic fuzzy rough sets. Information Sciences, 216, pp.34-49.

[29] Yun, S.M. and Lee, S.J., 2015. Intuitionistic fuzzy rough approximation operators. International Journal of Fuzzy Logic and Intelligent Systems, 15(3), pp.208-215.

[30] Chakrabarty, K., Gedeon, T. and Koczy, L., 1998. Intuitionistic fuzzy rough set. In Proceedings of 4th joint conference on information sciences (JCIS), Durham, NC (pp. 211-214).

[31] S. P. Jena, S. K. Ghosh, and B. K. Tripathy, "Intuitionistic fuzzy rough sets," Notes IFS., vol. 8, no. 1, pp. 1-18, 2002.

[32] Samanta, S.K. and Mondal, T.K., 2001. Intuitionistic fuzzy rough sets and rough intuitionistic fuzzy sets. Journal of Fuzzy Mathematics, 9(3), pp.561-582.

[33] Zhou, L., Wu, W.Z. and Zhang, W.X., 2009. On characterization of intuitionistic fuzzy rough sets based on intuitionistic fuzzy implicators. Information Sciences, 179(7), pp.883-898.

[34] Chinram, R., Hussain, A., Mahmood, T. and Ali, M.I., 2021. EDAS method for multicriteria group decision making based on intuitionistic fuzzy rough aggregation operators. IEEE Access, 9, pp.10199-10216.

[35] Hwang, C.L. and Yoon, K., 1981. Methods for multiple attribute decision making. In Multiple attribute decision making (pp. 58-191). Springer, Berlin, Heidelberg.

[36] Chen, C.T., 2000. Extensions of the TOPSIS for group decision-making under fuzzy environment. Fuzzy sets and systems, 114(1), pp.1-9.

[37] Beg, I. and Rashid, T., 2013. TOPSIS for hesitant fuzzy linguistic term sets. International Journal of Intelligent Systems, 28(12), pp.1162-1171.

[38] Biswas, A. and Kumar, S., 2018. An integrated TOPSIS approach to MADM with intervalvalued intuitionistic fuzzy settings. In Advanced Computational and Communication Paradigms (pp. 533-543). Springer,Singapore.

[39] Cables, E., García-Cascales, M.S. and Lamata, M.T., 2012. The L-TOPSIS: An alternative to TOPSIS decision-making approach for linguistic variables. Expert Systems with Applications, 39(2), pp.2119-2126.

[40] Chen, T.Y. and Tsao, C.Y., 2008. The interval-valued fuzzy TOPSIS method and experimental analysis. Fuzzy sets and systems, 159(11), pp.1410-1428.

[41] Li, D.F., 2010. TOPSIS-based nonlinear-programming methodology for multi-attribute decision making with interval-valued intuitionistic fuzzy sets. IEEE Transactions on Fuzzy Systems, 18(2), pp.299-311.

[42] Liang, D. and Xu, Z., 2017. The new extension of TOPSIS method for multiple criteria decision making with hesitant Pythagorean fuzzy sets. Applied Soft Computing, 60, pp.167179.

[43] Mohagheghi, V., Mousavi, S.M. and Vahdani, B., 2017. Enhancing decision-making flexibility by introducing a new last aggregation evaluating approach based on multi-criteria group decision making and Pythagorean fuzzy sets. Applied Soft Computing, 61, pp.527-535.

[44] Park, J.H., Park, I.Y., Kwun, Y.C. and Tan, X., 2011. Extension of the TOPSIS method for decision making problems under interval-valued intuitionistic fuzzy environment. Applied Mathematical Modelling, 35(5), pp.2544-2556. 
[45] Xu, Z. and Zhang, X., 2013. Hesitant fuzzy multi-attribute decision making based on TOPSIS with incomplete weight information. Knowledge-Based Systems, 52, pp.53-64.

[46] Yang, Y., Ding, H., Chen, Z.S. and Li, Y.L., 2016. A note on extension of TOPSIS to multiple criteria decision making with Pythagorean fuzzy sets. International Journal of Intelligent Systems, 31(1), pp.68-72.

[47] Zeng, S., Chen, J. and Li, X., 2016. A hybrid method for Pythagorean fuzzy multiple-criteria decision making. International Journal of Information Technology \& Decision Making, 15(02), pp.403-422.

[48] Zhang, X. and Xu, Z., 2014. Extension of TOPSIS to multiple criteria decision making with Pythagorean fuzzy sets. International Journal of Intelligent Systems, 29(12), pp.1061-1078.

[49] Boran, F.E., Genç, S., Kurt, M. and Akay, D., 2009. A multi-criteria intuitionistic fuzzy group decision making for supplier selection with TOPSIS method. Expert Systems with Applications, 36(8), pp.11363-11368.

[50] Shih, H.S., 2008. Incremental analysis for MCDM with an application to group TOPSIS. European Journal of Operational Research, 186(2), pp.720-734.

[51] Torlak, G., Sevkli, M., Sanal, M. and Zaim, S., 2011. Analyzing business competition by using fuzzy TOPSIS method: An example of Turkish domestic airline industry. Expert Systems with Applications, 38(4), pp.3396-3406.

[52] Yue, Z., 2011. An extended TOPSIS for determining weights of decision makers with interval numbers. Knowledge-Based Systems, 24(1), pp.146-153.

[53] Yue, Z., 2013. An avoiding information loss approach to group decision making. Applied Mathematical Modelling, 37(1-2), pp.112-126.

[54] Guo, K. and Song, Q., 2014. On the entropy for Atanassov's intuitionistic fuzzy sets: An interpretation from the perspective of amount of knowledge. Applied Soft Computing, 24, pp.328-340.

[55] Biswas, A. and Sarkar, B., 2019. Pythagorean fuzzy TOPSIS for multicriteria group decisionmaking with unknown weight information through entropy measure. International Journal of Intelligent Systems, 34(6), pp.1108-1128.

[56] Wang, W. and Liu, X., 2012. Intuitionistic fuzzy information aggregation using Einstein operations. IEEE Transactions on Fuzzy Systems, 20(5), pp.923-938.

[57] Seikh, M.R. and Mandal, U., 2019. Intuitionistic fuzzy Dombi aggregation operators and their application to multiple attribute decision-making. Granular Computing, pp.1-16.

[58] Keshavarz Ghorabaee, M., Zavadskas, E.K., Olfat, L. and Turskis, Z., 2015. Multi-criteria inventory classification using a new method of evaluation based on distance from average solution (EDAS). Informatica, 26(3), pp.435-451.

[59] Yue Z. An avoiding information loss approach to group decision making. Appl Math Modelling. 2013;37(1):112-126.

Department of Mathematics, Abdul Wali Khan University Mardan, KP, Pakistan

E-mail address: abbasqadir@awkum.edu.pk

Department of Combined Year, Umm Al-Qura University, Makkah, Saudi Arabia

E-mail address: naeemtazkeer@yahoo.com

Department of Mathematics, Abdul Wali Khan University Mardan, KP, Pakistan

E-mail address: saleemabdullah@awkum.edu.pk

University College of Jammuh, Umm Al-Qura University Makkah, Saudi Aribia

E-mail address: naghnmi@uqu.edu.sa 